\title{
Transverse-momentum-dependent parton distribution functions up to twist 4 for spin-1 hadrons
}

\author{
S. Kumano ${ }^{1,2}$ and Qin-Tao Song, \\ ${ }^{1}$ KEK Theory Center, Institute of Particle and Nuclear Studies, \\ High Energy Accelerator Research Organization (KEK), Oho 1-1, Tsukuba, Ibaraki 305-0801, Japan \\ ${ }^{2} J$-PARC Branch, KEK Theory Center, Institute of Particle and Nuclear Studies, KEK, and Theory Group, \\ Particle and Nuclear Physics Division, J-PARC Center, Shirakata 203-1, Tokai, Ibaraki 319-1106, Japan \\ ${ }^{3}$ School of Physics and Microelectronics, Zhengzhou University, Zhengzhou, Henan 450001, China
}

(Received 17 November 2020; accepted 23 December 2020; published 25 January 2021; corrected 6 May 2021)

\begin{abstract}
We show possible transverse-momentum-dependent parton distribution functions (TMDs) for spin-1 hadrons including twist- 3 and twist- 4 functions in addition to the leading twist- 2 ones by investigating all the possible decomposition of a quark correlation function in the Lorentz-invariant way. The Hermiticity and parity invariance are imposed in the decomposition; however, the time-reversal invariance is not used due to an active role of gauge links in the TMDs. Therefore, there exist time-reversal-odd functions in addition to the time-reversal even ones in the TMDs. We list all the functions up to twist-4 level because there were missing terms associated with the light cone vector $n$ in previous works on the twist- 2 part and there was no correlation-function study in the twist- 3 and twist- 4 parts for spin- 1 hadrons. We show that 40 TMDs exist in the tensor-polarized spin-1 hadron in twists 2-4. Some expressions of twist-2 structure functions are modified from previous derivations due to the new terms with $n$, and we find 30 new structure functions in twists 3 and 4 in this work. Since time-reversal-odd terms of the collinear correlation function should vanish after integrals over the partonic transverse momentum, we obtain new sum rules for the timereversal-odd structure functions, $\int d^{2} k_{T} g_{L T}=\int d^{2} k_{T} h_{L L}=\int d^{2} k_{T} h_{3 L L}=0$. In addition, we indicate that new transverse-momentum-dependent fragmentation functions exist in tensor-polarized spin-1 hadrons. The TMDs are rare observables to find explicit color degrees of freedom in terms of color flow, which cannot be usually measured because the color is confined in hadrons. Furthermore, the studies of TMDs enable us not only to find three-dimensional structure of hadrons, namely, hadron tomography including transverse structure, but also to provide unique opportunities for creating interesting interdisciplinary physics fields such as gluon condensates, color Aharonov-Bohm effect, and color entanglement. The tensor structure functions may not be easily measured in experiments. However, high-intensity facility such as the Thomas Jefferson National Accelerator Facility, the Fermilab Main Injector, and future accelerators like electron-ion collider may probe such observables. In addition, since the Nuclotron-based Ion Collider fAcility focuses on spin-1 deuteron structure functions, there is a possibility to study the details of polarized structure functions of the deuteron at this facility.
\end{abstract}

DOI: 10.1103/PhysRevD.103.014025

\section{INTRODUCTION}

It had been taken for granted that the proton spin consists of three quark spins in the naive quark model. However, the European Muon Collaboration experiment found that the quark contribution accounts for only 20\%-30\% of the proton spin [1], and the rest should be carried by

\footnotetext{
*songqintao@zzu.edu.cn
}

Published by the American Physical Society under the terms of the Creative Commons Attribution 4.0 International license. Further distribution of this work must maintain attribution to the author(s) and the published article's title, journal citation, and DOI. Funded by SCOAP. gluon-spin and partonic orbital-angular-momentum (OAM) contributions [2]. In order to figure out the partonic OAM contributions, one needs to study three-dimensional (3D) structure functions which include generalized parton distributions (GPDs) [3], generalized distribution amplitudes (GDAs or timelike GPDs) [4], and transverse-momentumdependent parton distributions (TMDs) [5].

The TMDs indicate the parton distributions as the function of the partonic transverse momentum $k_{T}$ in addition to the longitudinal momentum fraction $x$. The color flow appears explicitly in the TMDs, although it does not show up easily in other observables because of the color confinement. They have interesting application to other fields of physics, such as the gluon condensate [6], color 
Aharonov-Bohm effect [7,8], and color entanglement [9]. The TMD studies are new tools to investigate the color degrees of freedom and to explore new interdisciplinary fields beyond standard hadron physics.

The TMDs have been investigated for the spin- $1 / 2$ proton; however, spin-1 hadrons and nuclei such as the deuteron have new interesting aspects due to the spin-1 nature. In the charged lepton scattering, there are four collinear structure functions $b_{1-4}$ in the deuteron in addition to the ones for the nucleon $[10,11]$. Among them, the leading-twist functions are $b_{1}$ and $b_{2}$, which are related with each other by the Callan-Gross type relation $2 x b_{1}=$ $b_{2}$ in the scaling limit $Q^{2} \rightarrow \infty$. These structure functions are expressed by tensor-polarized parton distribution functions (PDFs). In addition, there is the gluon transversity distribution [12] in the leading twist. For $b_{1}$, there were measurements by the HERMES Collaboration [13]; however, the magnitude and $x$ dependence of $b_{1}$ are very different from conventional convolution calculations based on a standard deuteron model with $D$-state admixture $[14,15]$. Furthermore, the HERMES data indicated a finite sum $\int d x b_{1}(x)=[0.35 \pm 0.10$ (stat) \pm 0.18 (sys) $] \quad$ [13], which indicates a finite tensor-polarized antiquark distribution according to the parton-model sum rule [16] $\int d x b_{1}(x)=-\lim _{t \rightarrow 0} \frac{5}{24} t F_{Q}(t)+\sum_{i} e_{i}^{2} \int d x \delta_{T} \bar{q}_{i}(x)$, where $F_{Q}(t)$ is the electric quadrupole form factor of the hadron and $\delta_{T} \bar{q}_{i}$ is the tensor-polarized antiquark distribution. The first term vanishes, so that a finite sum of $b_{1}$ indicates a finite tensor-polarized antiquark distribution. The vanishing first term comes from the fact that the valence-quark number does not depend on the tensor polarization, whereas it depends on the flavor in the Gottfried sum (1/3) [17].

Since the finite $b_{1}$ sum indicates a new topic on tensorpolarized antiquark distributions and the standard convolution-model distribution for $b_{1}$ is very different from the HERMES data [14], a new high-energy spin physics field could be foreseen by investigating tensorpolarized structure functions. Experimentally, there is an experimental proposal to measure $b_{1}$ accurately measured at Thomas Jefferson National Accelerator Facility (JLab) [18] and tensor-polarized PDFs could be measured at Fermilab by the SpinQuest (E1039) experiment [19] by the proton-deuteron Drell-Yan (DY) process with the tensor-polarized deuteron target [20]. The proton-deuteron Drell-Yan formalism was given in Ref. [21], and tensorpolarized spin asymmetries were estimated in Ref. [22] based on the parametrization for the tensor-polarized PDFs of Ref. [23]. There were also GPD studies on the spin-1 deuteron and $\rho$ meson [24] and fragmentation-function studies on spin-1 hadrons [25].

On the gluon transversity, there is an experimental plan to measure it at JLab [12] and there is a possibility to study it at Fermilab by using the proton-deuteron DrellYan process with the linearly polarized deuteron [26].
Furthermore, there are possibilities at NICA (Nuclotronbased Ion Collider fAcility) [27] and GSI-FAIR (Gesellschaft für Schwerionenforschung-Facility for Antiproton and Ion Research). Since the spin- $1 / 2$ proton and neutron in the deuteron cannot contribute to the gluon transversity, it is an appropriate quantity to find new hadron physics beyond the simple bound system of the nucleons.

These $b_{1}$ and gluon transversity distribution are collinear functions as the function of $x$. In this work, we investigate possible TMDs for spin-1 hadrons especially by considering the tensor polarization. The TMDs are generally defined from the quark correlation function. The quark correlation function and its relations to PDFs were investigated for the spin-1/2 nucleon in Refs. [28,29], and additional terms were studied in Ref. [21] for the spin-1 deuteron. The quark TMD correlation function was decomposed into possible terms by considering Lorentz invariance, Hermiticity, and parity conservation in Refs. [30,31] for spin- $1 / 2$ proton, and then the TMDs were introduced by integrating the correlation function over the minus component of the quark light cone momentum.

Much progress has been made in the TMD studies based on these works; however, it was found later that the decomposition of the quark correlation function in Refs. [21,28,30,31] was not complete. The quark correlation function depends on the light cone vector $n$, which is defined in Eq. (4), due to the gauge link or the Wilson line $W(0, \xi \mid n)$ which guarantees the color gauge invariance of the correlation function [32-34] as defined later in Eqs. (2), (6), and (8). The vector $n$ specifies the direction along the gauge link. The complete decomposition of the quark correlation function was made by introducing 20 new terms which are associated with the light cone vector $n$ for the spin-1/2 nucleon in Ref. [34]. Even though these new terms in the correlation function do not give rise to new TMDs at the leading-twist level, they bring new observables in the semi-inclusive deep inelastic scattering (SIDIS) which are expressed by the new twist-3 TMDs [33]. The new terms in the correlation function also affect relations of the collinear PDFs. For example, several Lorentz invariance relations for the PDFs were obtained [35] based on the decomposition of the quark correlation function in Refs. [30,31], and these relations were modified if one considered the complete decomposition of the correlation function [32,36]. Moreover, the WandzuraWilczek relation [37] was reinvestigated in Refs. [38,39], it was found that the Wandzura-Wilczek relation was not satisfied due to another new twist-3 term.

These additional terms due to $n$ were studied for the spin$1 / 2$ nucleon [34]. The purpose of this work is to derive new TMDs associated with $n$ for spin- 1 hadrons up to the twist4 level. As for a stable spin-1 hadron or nucleus for experiments, the deuteron is the most simple and stable particle. It is known that there are additional structure functions in the spin-1 deuteron in comparison with the spin- $1 / 2$ nucleon, since both vector polarization and tensor 
polarization are available in the deuteron. The tensor polarization does not exist for the spin- $1 / 2$ nucleon, and it could be used to investigate new aspects in the deuteron. The deuteron is a weakly bound state of proton and neutron. However, the spin- $1 / 2$ proton and neutron do not contribute directly to the tensor structure, which is an interesting aspect in studying the deuteron's tensor polarizations.

As for theoretical studies, the spin-1 TMDs were investigated in Refs. [21,40,41] and T-even TMDs were calculated in an effective hadron model for the $\rho$ meson [42]. However, the terms associated with the light cone vector $n$ were not included in the decomposition of the quark-quark correlation function in Ref. [40]. Since these new terms could have a significant impact on the structure-function studies in the spin-1 hadrons, we show the complete decomposition of the quark correlation function for the spin-1 hadrons in this paper. In this paper, the transversemomentum-dependent quark correlation function and parton distribution functions are explained in Sec. II. Next, possible TMDs are obtained by decomposing the quark correlation function in Sec. III. Then, our studies are summarized in Sec. IV.

\section{TRANSVERSE-MOMENTUM-DEPENDENT PARTON DISTRIBUTION FUNCTIONS}

In this section, we introduce the TMDs and discuss motivations for investigating the TMDs. First, the threedimensional structure functions are explained as a field of hadron tomography from generalized TMDs and Wigner functions as generating functions in Sec. II A. The quark correlation function is introduced with proper gauge links, which play an important role in the TMD physics in Sec. II B. We show that the color flows, expressed by the gauge links, are different in the SIDIS and DY processes. This fact leads to the sign change in the time-reversal-odd quark TMDs. The time-reversal properties of the quark correlation function are discussed in Sec. II C.

\section{A. Hadron tomography by three-dimensional structure functions}

Until recently, hadron structure had been investigated by electromagnetic form factors and parton distribution functions (PDFs). However, recent studies focus on 3D aspects including the transverse structure in addition to the longitudinal one along the hadron-momentum direction. The 3D structure studies were originally motivated for finding the origin of nucleon spin including the partonic orbitalangular momenta (OAM). The OAM contribution to the nucleon spin should be probed by one of 3D structure functions, especially the GPDs. However, the hadron tomography, namely, the 3D structure of hadrons, has deeper meaning in the sense that it could probe gravitational form factors of hadrons without relying on explicit graviton interactions [4]. The hadron tomography has

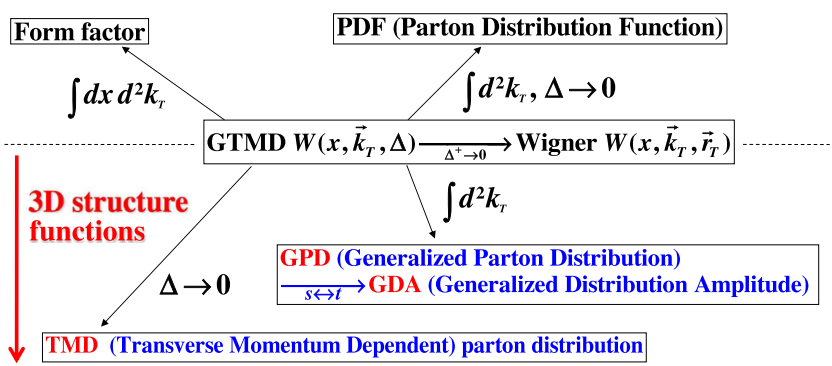

FIG. 1. Three-dimensional structure functions (TMD, GPD, GDA) from the generalized transverse-momentum-dependent parton distribution (GTMD) and the Wigner function, together with the form factor and parton distribution function.

been investigated by three types of structure functions, TMDs, GPDs, and GDAs (or timelike GPDs). They are obtained from the generating functions called generalized transverse-momentum-dependent parton distributions (GTMDs) and the Wigner functions as illustrated in Fig. 1. The TMDs are obtained by taking the forward limit $\Delta \rightarrow 0$, where $\Delta$ is the momentum transfer from the initial hadron to the final one $\left(\Delta=P^{\prime}-P\right)$, and the GPDs are obtained by integrating the GTMDs over the parton's transverse momentum $\vec{k}_{T}$. The GDAs are related to the GPDs by the $s-t$ crossing, where $s$ and $t$ are Mandelstam variables.

\section{B. Quark correlation functions and color flow}

The TMDs and collinear PDFs are defined from the quark correlation function

$$
\begin{aligned}
\Phi_{i j}^{[c]}(k, P, S, T)= & \int \frac{d^{4} \xi}{(2 \pi)^{4}} e^{i k \cdot \xi} \\
& \times\left\langle P, S, T\left|\bar{\psi}_{j}(0) W^{[c]}(0, \xi) \psi_{i}(\xi)\right| P, S, T\right\rangle,
\end{aligned}
$$

which is illustrated in Fig. 2. It may be denoted as $\Phi_{q / H, i j}^{[c]}$ with $q=u, d, s, \ldots$, but we abbreviated the notations on the quark flavor $q$ and the hadron $H$. The correlation function is related to the amplitude to extract a parton from a hadron and then to insert it into the hadron at a different spacetime point $\xi$. Here, $\psi$ is the quark field, $\xi$ is a four-dimensional space-time coordinate, $k$ and $P$ are the quark and hadron momenta, $S$ and $T$ are vector and tensor polarizations of the

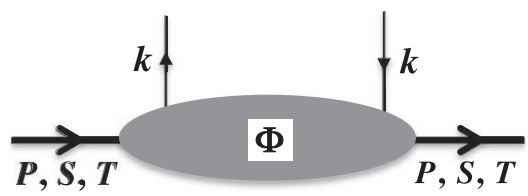

FIG. 2. Quark correlation function $\Phi$ with the quark (hadron) momentum $k(P)$ and the hadron vector and tensor polarizations $S$ and $T$, respectively. 
hadron, and $W^{[c]}(0, \xi)$ is called the gauge link or the Wilson line so as to satisfy the color gauge invariance. It is defined by the path-ordered exponential $(\mathcal{P})$

$$
W^{[c]}(0, \xi)=\mathcal{P} \exp \left[-i g \int_{0, c}^{\xi} d \xi \cdot A(\xi)\right]
$$

The gauge link indicates gluon interactions between quarks for satisfying the gauge invariance. Here, $c$ indicates the integral path, and $A_{\mu}(\xi)$ is $A_{\mu}(\xi)=A_{\mu}^{a}(\xi) t^{a}$ by including the SU(3) generator $t^{a}$ expressed by the Gell-Mann matrix $\lambda^{a}$ as $t^{a}=\lambda^{a} / 2$ with the color index $a$. The antiquark correlation function is defined in the same way [26].

The TMDs and collinear PDFs for quarks are then given by the quark correlation functions as [26]

$$
\begin{aligned}
\Phi^{[c]}\left(x, k_{T}, P, S, T\right)= & \int d k^{+} d k^{-} \Phi^{[c]}(k, P, S, T \mid n) \\
& \times \delta\left(k^{+}-x P^{+}\right), \\
\Phi(x, P, S, T)= & \int d^{2} k_{T} \Phi^{[c]}\left(x, k_{T}, P, S, T\right),
\end{aligned}
$$

where $k_{T}$ is the quark transverse momentum, $\Phi^{[c]}\left(x, k_{T}\right.$, $P, S, T)$ is the transverse-momentum-dependent correlation function which is related later to the TMDs, and $\Phi(x, P, S, T)$ is the collinear correlation function. The light cone \pm momenta are defined by $a^{ \pm}=\left(a^{0} \pm a^{3}\right) / \sqrt{2}$, and the light cone vectors $n$ and $\bar{n}$ are given by

$$
n^{\mu}=\frac{1}{\sqrt{2}}(1,0,0,-1), \quad \bar{n}^{\mu}=\frac{1}{\sqrt{2}}(1,0,0,1) .
$$

The integral path depends on the light cone direction $n^{-}$, which is explicitly shown as the $n$ dependence in Eq. (3). We note that there is no link-path dependence $c$ in the collinear correlation function $\Phi(x, P, S, T)$ as shown in this section. From Eqs. (1) and (3), the transverse-momentumdependent correlation function is expressed as

$$
\begin{aligned}
& \Phi_{i j}^{[c]}\left(x, k_{T}, P, S, T\right) \\
& =\int \frac{d \xi^{-} d^{2} \xi_{T}}{(2 \pi)^{3}} e^{i k^{+} \xi^{-}-i \vec{k}_{T} \cdot \vec{\xi}_{T}} \\
& \quad \times\left\langle P, S, T\left|\bar{\psi}_{j}(0) W^{[c]}(0, \xi \mid n) \psi_{i}(\xi)\right| P, S, T\right\rangle_{\xi^{+}=0},
\end{aligned}
$$

with the plus light cone momentum $k^{+}=x P^{+}$by taking the hadron momentum direction as the third axis.

The details of the gauge link for the SIDIS are, for example, explained in Ref. [43]. Resummations of processes with intermediate gluons, as typically shown in Fig. 3, lead to the gauge link [5,43-46]. Here, the gauge link $W^{[c]}(0, \xi \mid n)$ for the TMD correlation function in the SIDIS process $(c=+)$ is given by

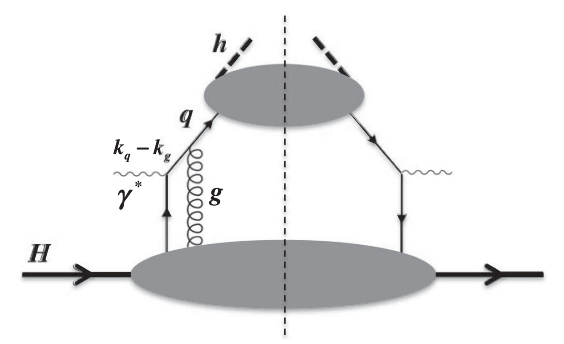

FIG. 3. A typical semi-inclusive DIS process $\gamma^{*}+H \rightarrow h+X$ $\left(\ell \rightarrow \gamma^{*} \ell^{\prime}, \ell=e\right.$ or $\mu$ ) with a gluon interaction in the final state.

$$
\begin{aligned}
W^{[+]}(0, \xi \mid n)= & {\left[0, \overrightarrow{0}_{T} ; \infty, \overrightarrow{0}_{T}\right]\left[\infty, \overrightarrow{0}_{T} ; \infty, \vec{\xi}_{T}\right] } \\
& \times\left[\infty, \vec{\xi}_{T} ; \xi^{-}, \vec{\xi}_{T}\right]_{\xi^{+}=0} .
\end{aligned}
$$

Here, the notation $\left[a^{-}, \vec{a}_{T} ; b^{-}, \vec{b}_{T}\right]$ (or doted as $W(a, b \mid n)$ ) indicates the gauge line connecting $a=\left(a^{+}=0, a^{-}, \vec{a}_{T}\right)$ to $b=\left(b^{+}=0, b^{-}, \vec{b}_{T}\right)$ along the straight light cone direction of $\xi^{-}$(namely, plus direction of $n^{-}$), and $\left[a^{-}, \vec{a}_{T} ; b^{-}, \vec{b}_{T}\right]\left(W\left(a, b \mid \vec{\xi}_{T}\right)\right)$ is the link along the transverse direction $\vec{\xi}_{T}$,

$$
\begin{aligned}
W(a, b \mid n) & =\left[a^{-}, \vec{a}_{T} ; b^{-}, \vec{b}_{T}\left(=\vec{a}_{T}\right)\right] \\
& \equiv \mathcal{P} \exp \left[-i g \int_{a^{-}}^{b^{-}} d \xi^{-} A^{+}(\xi)\right]_{\substack{\xi^{+}=a^{+}=b^{+} \\
\xi_{T}=\vec{a}_{T}=b_{T}}}, \\
W\left(a, b \mid \vec{\xi}_{T}\right) & =\left[a^{-}, \vec{a}_{T} ; b^{-}\left(=a^{-}\right), \vec{b}_{T}\right] \\
& \equiv \mathcal{P} \exp \left[-i g \int_{\vec{a}_{T}}^{\vec{b}_{T}} d \vec{\xi}_{T} \cdot \vec{A}_{T}(\xi)\right]_{\xi^{ \pm}=a^{ \pm}=b^{ \pm}} .
\end{aligned}
$$

The superscript $[+]$ of $W^{[+]}$in Eq. (6) indicates the integral path along the plus direction in the coordinate $\xi^{-}$in the first link step. The final expression for the link path of Eq. (6) is shown in $(a)$ of Fig. 4 . The path $c=+$ consists of the three gauge links. The path dependence of the gauge link is important in TMD physics, as we show the difference between the TMDs of the SIDIS and the Drell-Yan process in the following.

A typical Drell-Yan process $H_{1}+H_{2} \rightarrow \gamma^{*}+X$ $\left(\gamma^{*} \rightarrow \mu^{-} \mu^{+}\right)$with an intermediate gluon is shown in Fig. 5. We note that the gluon exchange occurs in the initial state, whereas it does in the final state in the SIDIS [47] as shown in Fig. 3. It leads to the path difference in the

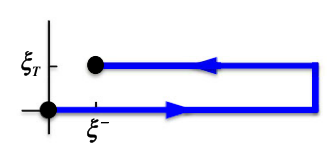

(a)

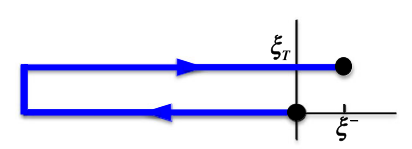

(b)
FIG. 4. Gauge link for (a) semi-inclusive DIS with the spacelike correlation function $\Phi^{[+]}$and (b) Drell-Yan process with the timelike correlation function $\Phi^{[-]}$. 


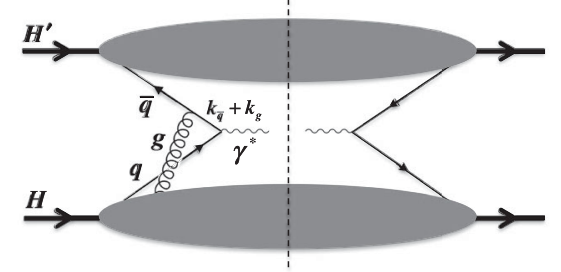

FIG. 5. A typical Drell-Yan process $H+H^{\prime} \rightarrow \gamma^{*}+X$ $\left(\gamma^{*} \rightarrow \mu^{-} \mu^{+}\right)$with a gluon interaction in the initial state.

gauge link and subsequently in the sign difference in both TMDs. The cross sections of these SIDIS and Drell-Yan processes are calculated $[5,43,46]$, and it was found that the color flows in the opposite light cone direction between the SIDIS and Drell-Yan processes. Therefore, the gauge link for the Drell-Yan process $(c=-)$ is given by

$$
\begin{aligned}
W^{[-]}(0, \xi \mid n)= & {\left[0, \overrightarrow{0}_{T} ;-\infty, \overrightarrow{0}_{T}\right]\left[-\infty, \overrightarrow{0}_{T} ;-\infty, \vec{\xi}_{T}\right] } \\
& \times\left[-\infty, \vec{\xi}_{T} ; \xi^{-}, \vec{\xi}_{T}\right]_{\xi^{+}=0},
\end{aligned}
$$

as shown in Fig. 4(b). We notice that the gauge links of the SIDIS and Drell-Yan are opposite in the $\xi^{-}$direction, which results in the sign change in the time-reversal-odd TMDs as shown in Eq. (16). The superscript [-] indicates the integral path of the first link step along the minus direction in the coordinate $\xi^{-}$.

If the transverse momentum is integrated as in Eq. (3), the collinear correlation function of Eq. (5) becomes

$$
\begin{aligned}
& \Phi_{i j}(x, P, S, T) \\
& =\int \frac{d \xi^{-}}{2 \pi} e^{i x P^{+} \xi^{-}} \\
& \quad \times\left\langle P, S, T\left|\bar{\psi}_{j}(0) W(0, \xi \mid n) \psi_{i}(\xi)\right| P, S, T\right\rangle_{\xi^{+}=0, \vec{\xi}_{T}=0},
\end{aligned}
$$

where $k^{+}=x P^{+}$is used. Since the gauge link is the straight line from $\xi^{-}=0$ to $\xi^{-}$with $\xi^{+}=0$ and $\vec{\xi}_{T}=0$, these collinear functions are not dependent on the gaugelink path like the TMDs and a process-dependent relation like Eq. (16) does not exist. The color flow can be probed only if transverse-momentum-dependent observables are investigated.

\section{Existence of time-reversal-odd structure functions and their color-flow dependence}

Here, we show properties of the TMD correlation function under the time reversal for defining time reversal even and odd functions. However, one should note that the existence of the time-reversal-odd functions does not mean the violation of the time-reversal invariance as explained in this subsection.

The parity and time reversal mean the transformations of the space-time coordinate as

$$
\begin{aligned}
\text { Parity: } x^{\mu}=(t, \vec{x}) \rightarrow(t,-\vec{x}), \\
\text { Time reversal: } x^{\mu}=(t, \vec{x}) \rightarrow(-t, \vec{x}) .
\end{aligned}
$$

The parity $(\mathscr{P})$ is a unitary operator and the time-reversal $(\mathscr{T})$ is an antiunitary one $[48,49]$. The antiunitary means antilinear with the unitarity. Namely, it satisfies the relations

$$
\text { Antilinear: } \mathscr{T}(a|A\rangle+b|B\rangle)=a^{*} \mathscr{T}|A\rangle+b^{*} \mathscr{T}|B\rangle,
$$

Hermite conjugate: $\left\langle A\left|\mathscr{T}^{\dagger}\right| B\right\rangle=\langle\mathscr{T} A \mid B\rangle^{*}$,

where the definition of the Hermite conjugate is different from the usual definition $\left\langle A\left|\mathcal{O}^{\dagger}\right| B\right\rangle=\langle\mathcal{O} A \mid B\rangle$ for the linear operator $\mathcal{O}$. The momentum $(P)$, spin $(S)$, and tensor $(T)$ transform under parity and time-reversal transformations as shown in Table I, where $\bar{P}^{\mu}$ and $\bar{T}^{\mu \nu}$ are defined by

$$
\bar{P}^{\mu} \equiv\left(P^{0},-\vec{P}\right)=g^{\mu \alpha} P_{\alpha}, \quad \bar{T}^{\mu \nu}=g^{\mu \alpha} g^{\nu \beta} T_{\alpha \beta} .
$$

Under the parity and time reversal, the transformations of the quark field $\psi(\xi)$ and the gluon field $A_{\mu}(\xi)[43,50,51]$ are

TABLE I. Properties under Hermite, parity, and time-reversal transformations. The spin $S$, tensor $T$, and light cone vector $n$ are abbreviated in $\Phi^{[ \pm]}\left(x, k_{T}\right)$ for simplicity because their transformations are shown in $\Phi(k, P, S, T \mid n)$. The charge conjugation is $C=i \gamma^{2} \gamma^{0}$ so that the time-reversal factor is $\mathcal{T}=-i \gamma_{5} C=i \gamma^{1} \gamma^{3}$. The time-reversal invariance condition is not imposed for the correction functions due to the gauge link; however, we show the time-reversal properties in this table to understand the T-even and odd properties in our formalism.

\begin{tabular}{lccc}
\hline \hline Quantity & Hermite & Parity & Time reversal \\
\hline$P^{\mu}$ & & $\bar{P}^{\mu}$ & $\bar{P}^{\mu}$ \\
$S^{\mu}$ & $-\bar{S}^{\mu}$ & $\bar{S}^{\mu}$ \\
$T^{\mu \nu}$ & $\bar{T}^{\mu \nu}$ & $\bar{T}^{\mu \nu}$ \\
$\psi(\xi)$ & $\mathscr{P} \psi(\xi) \mathscr{P}^{\dagger}=\gamma^{0} \psi(\bar{\xi})$ & $\mathscr{T} \psi(\xi) \mathscr{T}^{\dagger}=\left(-i \gamma_{5} C\right) \psi(-\bar{\xi})$ \\
$A_{\mu}(\xi)$ & $\mathscr{P} A_{\mu}(\xi) \mathscr{P}^{\dagger}=\bar{A}_{\mu}(\bar{\xi})$ & $\mathscr{T} A_{\mu}(\xi) \mathscr{T}^{\dagger}=\bar{A}_{\mu}(-\bar{\xi})$ \\
$W(a, b)$ & $A_{\mu}^{\dagger}(\xi)=A_{\mu}(\xi)$ & $\mathscr{T} W(a, b) \mathscr{T}^{\dagger}=W(-\bar{a},-\bar{b})$ \\
$\Phi(k, P, S, T \mid n)$ & $\Phi^{\dagger}(k, P, S, T \mid n)=\gamma^{0} \Phi(k, P, S, T \mid n) \gamma^{0}$ & $\Phi(k, P, S, T \mid n)=\gamma^{0} \Phi(\bar{k}, \bar{P},-\bar{S}, \bar{T} \mid \bar{n}) \gamma^{0}$ & $\Phi^{*}(k, P, S, T \mid n)=\left(-i \gamma_{5} C\right) \Phi(\bar{k}, \bar{P}, \bar{S}, \bar{T} \mid \bar{n})\left(-i \gamma_{5} C\right)$ \\
$\Phi^{[ \pm]}\left(x, k_{T}\right)$ & $\Phi^{[ \pm \dagger}\left(x, k_{T}\right)=\gamma^{0} \Phi^{[ \pm]}\left(x, k_{T}\right) \gamma^{0}$ & $\Phi^{ \pm \pm]}\left(x, k_{T}\right)=\gamma^{0} \Phi^{ \pm \pm]}\left(x, \bar{k}_{T}\right) \gamma^{0}$ & $\Phi^{[ \pm \pm *}\left(x, k_{T}\right)=\left(-i \gamma_{5} C\right) \Phi^{[\mp]}\left(x, \bar{k}_{T}\right)\left(-i \gamma_{5} C\right)$ \\
\hline \hline
\end{tabular}


shown in Table I, together with the Hermite conjugation for $A_{\mu}(\xi)$. Here, the charge conjugation $C$ is given by $C=i \gamma^{2} \gamma^{0}$, so that the overall factor is $\mathcal{T}=-i \gamma_{5} C=i \gamma^{1} \gamma^{3}$.

From the transformations of the gluon field, the gauge link $W(a, b)$ should have the transformations in Table I. These relations mean that the link paths are changed due to the space-time coordinate changes $a, b \rightarrow \bar{a}, \bar{b}$ (or $-\bar{a},-\bar{b}$ ) $[43,52]$. The $\gamma^{0}$ factors, for example, in the Hermiticity relation are obtained simply by taking the Hermite conjugate of the correlation function in Eq. (1). The transformations for the TMD correlation function $\Phi^{[ \pm]}\left(x, k_{T}\right)$ are then given in Table I, so that the time reversal transforms $\Phi^{[+]}$to $\Phi^{[-]}$and vice versa. The T-even and T-odd TMD functions are then defined by

$$
\begin{aligned}
\Phi^{[\text {T-even }]}\left(x, k_{T}\right) & =\frac{1}{2}\left[\Phi^{[+]}\left(x, k_{T}\right)+\Phi^{[-]}\left(x, k_{T}\right)\right], \\
\Phi^{[\text {T-even }]}\left(x, k_{T}\right) & =\frac{1}{2}\left[\Phi^{[+]}\left(x, k_{T}\right)-\Phi^{[-]}\left(x, k_{T}\right)\right] .
\end{aligned}
$$

If the transverse moment $\Phi^{[ \pm] \mu}(x)$ is defined by

$$
\Phi_{\partial}^{[ \pm] \mu}(x)=\int d^{2} k_{T} k_{T}^{\mu} \Phi^{[ \pm]}\left(x, k_{T}\right),
$$

they are given by the T-odd quark-gluon correlation function $\Phi_{G}^{[\text {T-odd }] \mu}(x, x)$ as [43]

$$
\Phi_{\partial}^{[ \pm] \mu}(x)=\Phi_{\partial}^{[\text {T-even }] \mu}(x) \pm \pi \Phi_{G}^{[\mathrm{T}-\text { odd }]} \mu(x, x) .
$$

These different link paths give rise to sign differences in the time-reversal-odd TMD functions. The second term of this equation $\left(\Phi_{G}^{[\mathrm{T}-\mathrm{odd}] \mu}\right)$ comes from the soft gluon $\left(k_{g}^{+}=0\right)$ and it is called gluonic-pole matrix. It suggests that the single spin asymmetries, such as the Sivers effect, originate from this term, as proposed by Qiu and Sterman [53]. Here, the Sivers function is one of the TMDs and it indicates the difference between a unpolarized quark distribution in the nucleon polarized transversely to its momentum and the one with opposite polarization. The T-odd TMDs exist in the single spin asymmetries in SIDIS by the form $\Phi_{\partial}^{[+] \mu}(x)$ and in the Drell-Yan by $\Phi_{\partial}^{[-] \mu}(x)$ [43]. This fact leads to the sign change in the T-odd quark TMD functions [54],

$$
f_{\text {SIDIS }}\left(x, k_{T}^{2}\right)_{\mathrm{T} \text {-odd }}=-f_{\mathrm{DY}}\left(x, k_{T}^{2}\right)_{\mathrm{T} \text {-odd }} .
$$

The difference comes from the fact that the color interactions are in the final state for the SIDIS and in the initial state for the Drell-Yan as shown in Figs. 3 and 5, respectively. It leads to the difference on the color-flow path between Eqs. (6) and (8).

The color is confined in hadrons, so that the color degrees of freedom usually does not appear explicitly in physical observables. However, depending on the color-flow direction, the T-odd TMDs are different in sign. The TMD case is a rare and special occasion to investigate the color flow, namely, the color degrees of freedom, in hadron physics. It was predicated theoretically that the TMDs are different in sign between the SIDIS and the DrellYan process. In fact, there are already experimental indications on this new phenomenon in the Sivers functions. About the experimental signatures on the sign change in the TMDs, it was suggested in the spin asymmetry of the reaction $\vec{p}+p \rightarrow W^{ \pm} / Z^{0}+X$ by the STAR Collaboration [55] and the spin asymmetry of $\pi^{-}+$ $\vec{p} \rightarrow \mu^{+} \mu^{-}+X$ by the COMPASS Collaboration [56]. Further confirmations on these effects are needed by future accurate experiments.

\section{RESULTS ON TMDS FOR TENSOR-POLARIZED SPIN-1 HADRONS}

We derive possible quark TMDs for tensor-polarized spin-1 hadrons in this section by the decomposition of the quark correlation function in terms of kinematical factors in the Lorentz-invariant manner. In particular, we find new terms associated with the light cone vector $n$ in this work. First, we try to obtain all the possible terms in the expansion of the quark TMD correlation function in Sec. III A by considering a tensor-polarized spin-1 hadron. Then, properties of each expansion term are discussed on Hermiticity, parity, time reversal, chirality, and twist in Sec. III B. Next, our guideline is explained for assigning various TMD notations in Sec. III C, and we show possible twist-2, 3, and 4 quark TMDs in Secs. III D, III E, and III F, respectively. A brief summary is given on the new TMDs and possible new fragmentation functions are explained in Sec. III G. The new terms associated with $n$ modify the relations in the twist-2 TMDs, which were obtained in the previous work [40]. In addition, we show that there are new twist-3 and 4 TMDs in this work.

\section{A. Decomposition of quark correlation function}

For spin- $1 / 2$ nucleon, the spin density matrix is parametrized with the spin vector which contains three parameters. However, due to the spin-1 nature, the spin density matrix of the spin-1 hadron, such as the deuteron, is determined by spin tensor in addition to the spin vector. There are five parameters in the spin tensor part, and the spin-vector part of spin-1 hadron is the same as the one of the spin- $1 / 2$ nucleon.

For expressing polarizations of the spin- 1 hadron, its density matrix is given by spin vector and tensor terms as $[26,40]$

$$
\boldsymbol{\rho}=\frac{1}{3}\left(1+\frac{3}{2} S_{i} \boldsymbol{\Sigma}_{i}+3 T_{i j} \boldsymbol{\Sigma}_{i j}\right) .
$$


Here, $\boldsymbol{\Sigma}_{i}$ are $3 \times 3$ spin matrices for the spin-1 hadron, and $\boldsymbol{\Sigma}_{i j}$ are spin tensors defined by $\boldsymbol{\Sigma}_{i j}=\left(\boldsymbol{\Sigma}_{i} \boldsymbol{\Sigma}_{j}+\boldsymbol{\Sigma}_{j} \boldsymbol{\Sigma}_{i}\right) / 2-$ $(2 / 3) \boldsymbol{I} \delta_{i j}$ with the $3 \times 3$ identity matrix $\boldsymbol{I}$. The spin vector and tensor are parametrized as

$\boldsymbol{S}=\left(S_{T}^{x}, S_{T}^{y}, S_{L}\right)$,

$\boldsymbol{T}=\frac{1}{2}\left(\begin{array}{ccc}-\frac{2}{3} S_{L L}+S_{T T}^{x x} & S_{T T}^{x y} & S_{L T}^{x} \\ S_{T T}^{x y} & -\frac{2}{3} S_{L L}-S_{T T}^{x x} & S_{L T}^{y} \\ S_{L T}^{x} & S_{L T}^{y} & \frac{4}{3} S_{L L}\end{array}\right)$

in the rest frame of the spin-1 hadron. The parameters $S_{T}^{x}$ and $S_{T}^{y}$ indicate transverse polarizations of the hadron, and $S_{L}$ does the longitudinal polarization. The parameter $S_{L L}$ indicates the tensor polarization along the longitudinal axis as shown in Ref. [40], and $S_{L T}^{x, y}\left(S_{T T}^{x x, x y}\right)$ indicate polarization differences along the axes between the longitudinal and transverse directions (along the transverse axes). The linear polarizations are parts of the tensor polarizations. These tensor and linear polarizations are schematically shown in the Appendix of Ref. [40].

The covariant forms of $S^{\mu}$ and $T^{\mu \nu}$ of a spin-1 hadron are generally expressed as $[22,40]$

$$
\begin{aligned}
S^{\mu}= & S_{L} \frac{P^{+}}{M} \bar{n}^{\mu}-S_{L} \frac{M}{2 P^{+}} n^{\mu}+S_{T}^{\mu}, \\
T^{\mu \nu}= & \frac{1}{2}\left[\frac{4}{3} S_{L L} \frac{\left(P^{+}\right)^{2}}{M^{2}} \bar{n}^{\mu} \bar{n}^{\nu}-\frac{2}{3} S_{L L}\left(\bar{n}^{\{\mu} n^{\nu\}}-g_{T}^{\mu \nu}\right)\right. \\
& +\frac{1}{3} S_{L L} \frac{M^{2}}{\left(P^{+}\right)^{2}} n^{\mu} n^{\nu}+\frac{P^{+}}{M} \bar{n}^{\{\mu} S_{L T}^{\nu\}} \\
& \left.-\frac{M}{2 P^{+}} n^{\{\mu} S_{L T}^{\nu\}}+S_{T T}^{\mu \nu}\right],
\end{aligned}
$$

where $a^{\{\mu} b^{\nu\}}$ indicates the symmetrized combination $a^{\{\mu} b^{\nu\}}=a^{\mu} b^{\nu}+a^{\nu} b^{\mu}$, and $M$ is the hadron mass.

The general expression of the correlation function $\Phi(k, P, S, T)$ contains three parts: unpolarized, vectorpolarized, and tensor-polarized terms. The unpolarized and vector-polarized distributions in the spin-1 hadron are exactly the same as the relevant ones in the spin- $1 / 2$ nucleon; however, we briefly explain past studies on the quark correlation function for the nucleon. First, the quark correlation function was decomposed into nine terms by imposing Hermiticity, parity invariance, and time-reversal invariance in Ref. [29]. Then, the quark TMD correlation function was decomposed in Refs. [30,31] by introducing T-odd terms, and there are 12 terms with coefficients denoted as $A_{1}-A_{12}$. This decomposition was constructed with the vectors $P, S$, and $k$.

However, this decomposition was not complete because the quark correlation function depends on the vector $n$ through the gauge link $W(0, \xi \mid n)$. Therefore, the additional terms which depend on $n$ were investigated in Refs. [32-34,38,39], and 20 new terms were found and they are denoted as $B_{1}-B_{20}$. Therefore, there are 32 terms in total for the quark correlation function in the spin- $1 / 2$ nucleon. These new terms of $n$ are important for understanding all the TMDs, collinear PDFs, and their relations. Relations among the PDFs were derived by using the Lorentz invariant decomposition of the correlation function, so that they were often called "Lorentz-invariance relations" [35]. These relations were modified due to the existence of these new terms [32,36]. Furthermore, another new twist- 3 term appeared and it invalidated the WandzuraWilczek relation of the twist-2 level $[38,39]$. On the other hand, these new terms also introduced new TMDs such as $e_{T}^{\perp}\left(x, k_{T}^{2}\right), f_{T}^{\perp}\left(x, k_{T}^{2}\right)$, and $g^{\perp}\left(x, k_{T}^{2}\right)$ for the nucleon [32-34, 38]. The unpolarized and vector polarized terms in the quark correlation function of the spin-1 hadron are the same as the ones in the nucleon, and these 32 terms had been already studied [32,38].

In this work, we focus on the tensor-polarized part which does not exist in the spin-1/2 nucleon. The quark TMD correlation function of a spin-1 hadron was investigated in Ref. [21] by adding T-even terms to the nine terms in the nucleon case [29]. The T-odd terms should be also considered together with proper tensor polarizations [40], so that there are eight new terms in total in the tensor part, where the relevant coefficients were named as $A_{13}-A_{20}$. On the collinear PDFs of a spin-1 hadron, there are also studies in possible hadron-tensor terms, helicity amplitudes, and operator forms [11,12,57].

However, the terms with the vector $n$, which are found for the spin-1/2 nucleon, need to be added also in the formalism of the spin-1 hadron, namely, in the tensorpolarization part. We formulate these new terms in this work to find possible TMDs. Including these $n$ terms, we express the tensor part of quark correlation function $\Phi(k, P, T \mid n)$ for the spin-1 hadron as

$$
\begin{aligned}
\Phi(k, P, T \mid n)= & \frac{A_{13}}{M} T_{k k}+\frac{A_{14}}{M^{2}} T_{k k} \not P+\frac{A_{15}}{M^{2}} T_{k k} \not k+\frac{A_{16}}{M^{3}} \sigma_{P k} T_{k k}+A_{17} T^{k \nu} \gamma_{\nu}+\frac{A_{18}}{M} \sigma_{\nu P} T^{k \nu}+\frac{A_{19}}{M} \sigma_{\nu k} T^{k \nu} \\
& +\frac{A_{20}}{M^{2}} \varepsilon^{\mu \nu P k} \gamma_{\mu} \gamma_{5} T_{\nu k}+\frac{B_{21} M}{P \cdot n} T_{k n}+\frac{B_{22} M^{3}}{(P \cdot n)^{2}} T_{n n}+\frac{B_{23}}{P \cdot n M} \varepsilon^{\mu k P n} T_{\mu k}\left(i \gamma_{5}\right)+\frac{B_{24} M}{(P \cdot n)^{2}} \varepsilon^{\mu k P n} T_{\mu n}\left(i \gamma_{5}\right)+\frac{B_{25}}{P \cdot n} \not h T_{k k} \\
& +\frac{B_{26} M^{2}}{(P \cdot n)^{2}} \not h T_{k n}+\frac{B_{27} M^{4}}{(P \cdot n)^{3}} \not h T_{n n}+\frac{B_{28}}{P \cdot n} \not P T_{k n}+\frac{B_{29} M^{2}}{(P \cdot n)^{2}} \not P T_{n n}+\frac{B_{30}}{P \cdot n} \not k T_{k n}+\frac{B_{31} M^{2}}{(P \cdot n)^{2}} \not k T_{n n}+\frac{B_{32} M^{2}}{P \cdot n} \gamma_{\mu} T^{\mu n}
\end{aligned}
$$




$$
\begin{aligned}
& +\frac{B_{33}}{P \cdot n} \varepsilon^{\mu \nu P k} \gamma_{\mu} \gamma_{5} T_{\nu n}+\frac{B_{34}}{P \cdot n} \varepsilon^{\mu \nu P n} \gamma_{\mu} \gamma_{5} T_{\nu k}+\frac{B_{35} M^{2}}{(P \cdot n)^{2}} \varepsilon^{\mu \nu P n} \gamma_{\mu} \gamma_{5} T_{\nu n}+\frac{B_{36}}{P \cdot n M^{2}} \varepsilon^{\mu k P n} \gamma_{\mu} \gamma_{5} T_{k k} \\
& +\frac{B_{37}}{(P \cdot n)^{2}} \varepsilon^{\mu k P n} \gamma_{\mu} \gamma_{5} T_{k n}+\frac{B_{38} M^{2}}{(P \cdot n)^{3}} \varepsilon^{\mu k P n} \gamma_{\mu} \gamma_{5} T_{n n}+\frac{B_{39}}{(P \cdot n)^{2}} \not h \gamma_{5} T_{\mu k} \varepsilon^{\mu k P n}+\frac{B_{40} M^{2}}{(P \cdot n)^{3}} \not h \gamma_{5} T_{\mu n} \varepsilon^{\mu k P n} \\
& +\frac{B_{41}}{P \cdot n M} \sigma_{P k} T_{k n}+\frac{B_{42} M}{(P \cdot n)^{2}} \sigma_{P k} T_{n n}+\frac{B_{43}}{P \cdot n M} \sigma_{P n} T_{k k}+\frac{B_{44} M}{(P \cdot n)^{2}} \sigma_{P n} T_{k n}+\frac{B_{45} M^{3}}{(P \cdot n)^{3}} \sigma_{P n} T_{n n}+\frac{B_{46}}{P \cdot n M} \sigma_{k n} T_{k k} \\
& +\frac{B_{47} M}{(P \cdot n)^{2}} \sigma_{k n} T_{k n}+\frac{B_{48} M^{3}}{(P \cdot n)^{3}} \sigma_{k n} T_{n n}+\frac{B_{49} M}{P \cdot n} \sigma_{\mu n} T^{\mu k}+\frac{B_{50} M^{3}}{(P \cdot n)^{2}} \sigma_{\mu n} T^{\mu n}+\frac{B_{51} M}{P \cdot n} \sigma_{\mu P} T^{\mu n}+\frac{B_{52} M}{P \cdot n} \sigma_{\mu k} T^{\mu n},
\end{aligned}
$$

where the notation $X_{\mu k} \equiv X_{\mu \nu} k^{\nu}$ is used for brevity with the tensor $X$ as $\sigma^{\mu \nu}=i\left[\gamma^{\mu}, \gamma^{\nu}\right] / 2, T^{\mu \nu}$, or the antisymmetric tensor $\varepsilon^{\mu \nu \alpha \beta}$, and $k$ could be replaced by $n$ or $P$. We listed only the tensor terms proportional to the tensor polarization $T^{\mu \nu}$ in Eq. (19). Here, we use the convention $\varepsilon^{0123}=+1$ so as to agree with expressions in Ref. [40]. In deriving this equation, the Hermiticity and parity-invariance relations in Table I are imposed for the correlation function; however, the time-reversal invariance is not a necessary condition due to the existence of the gauge link.

The first eight terms $\left(A_{13}-A_{20}\right)$ were already obtained in Ref. [40], and they generated all the leading-twist TMDs. There are 40 terms in the tensor part of the quark correlation function, and 32 of them $\left(B_{21}-B_{52}\right)$ are dependent on the vector $n$. Therefore, the new terms, which we found in this work, are these 32 terms $B_{21}-B_{52}$.

In general, the coefficients $A_{i}(i=1-20)$ and $B_{i}$ $(i=1-52)$ depend on the scalars $k \cdot P, k^{2}, P \cdot n$, and $k \cdot n$. In order to keep $\Phi(k, P, T \mid n)$ invariant when the vector $n$ is replaced by $\lambda n$ as a scale change, $A_{i}$ and $B_{i}$ should be functions of $k^{2}$ and the ratios, $k \cdot n / P \cdot n$ and $k \cdot P$ [39]. The quark and hadron momenta $k$ and $P$ are expressed by two lightlike vectors $n$ and $\bar{n}$ as

$$
\begin{aligned}
P^{\mu} & =P^{+} \bar{n}^{\mu}+\frac{M^{2}}{2 P^{+}} n^{\mu}, \\
k^{\mu} & =x P^{+} \bar{n}^{\mu}+\frac{M^{2}(\sigma-x)}{2 P^{+}} n^{\mu}+k_{T}^{\mu},
\end{aligned}
$$

where $k_{T}^{2}\left(=-\vec{k}_{T}^{2}\right), \sigma$, and $\tau$ are given by

$$
k_{T}^{2}=\left(\tau+x^{2}-x \sigma\right) M^{2}, \quad \sigma \equiv \frac{2 k \cdot P}{M^{2}}, \quad \tau \equiv \frac{k^{2}}{M^{2}} .
$$

Here, $x$ is the light cone momentum fraction carried by the quark.

The $k_{T}$-dependent correlation function is obtained by integrating $\Phi(k, P, T \mid n)$ over $k^{-}$,

$$
\Phi\left(x, k_{T}, T\right)=\int d k^{-} \Phi(P, k, T \mid n)
$$

The TMD correlation function $\Phi\left(x, k_{T}, T\right)$ is used to describe the hard processes such as the semi-inclusive DIS and Drell-Yan process. Using the TMD correlation function of Eq. (23), we define the trace of TMD function by

$$
\Phi^{[\Gamma]}\left(x, k_{T}, T\right) \equiv \frac{1}{2} \operatorname{Tr}\left[\Phi\left(x, k_{T}, T\right) \Gamma\right],
$$

where $\Gamma$ is a gamma matrix. We reiterate that this correction function is only for the tensor-polarization $(T)$ part, and the unpolarized and vector-polarized $(S)$ terms are not included because they have been already investigated in previous works [34,38].

\section{B. Properties of Hermiticity, parity, time reversal, chirality, and twist}

Each term of the expansion in Eq. (20) satisfies the Hermiticity and parity invariance in Table I. The timereversal invariance is not imposed because of the active role of the gauge link in the TMDs. We explain the details on the conditions of Hermiticity, parity invariance, time-reversal invariance, chirality, and twist in the following.

\section{Hermiticity}

The Hermiticity condition $\Phi^{\dagger}(V, A, T)=\gamma^{0} \Phi(V, A, T) \gamma^{0}$, where $V$ is a Lorentz vector, $A$ is an axial vector, and $T$ is a tensor, is satisfied because of the relations $(\Gamma)^{\dagger}=\gamma^{0} \Gamma \gamma^{0}$ by taking $\Gamma$ as

$$
\mathbf{1}, \gamma^{\mu}, \gamma^{\mu} \gamma_{5}, i \gamma_{5}, \sigma^{\mu \nu}
$$

where 1 is the $4 \times 4$ identity matrix.

\section{Parity invariance}

The parity-invariance relation indicates $\Phi(V, A, T)=$ $\gamma^{0} \Phi(\bar{V},-\bar{A}, \bar{T}) \gamma^{0}$, which is satisfied, for example, because of the relation $\gamma^{0} \bar{V} \gamma^{0}=\varnothing$ for the vector $V^{\mu}$ and $\gamma^{0}\left(-\gamma_{5} \bar{A}\right) \gamma^{0}=\gamma_{5} \not \mathcal{A}$ for the axial vector $A^{\mu}$. We may note that the term $\varepsilon^{\mu X Y Z}=\varepsilon^{\mu \nu \alpha \beta} X_{\nu} Y_{\alpha} Z_{\beta}$ is an axial vector, so that $\gamma_{5} \gamma_{\mu} \varepsilon^{\mu X Y Z}$ and $i \gamma_{5} \varepsilon^{V X Y Z}$ terms satisfy the parity invariance. Here, $X, Y$, and $Z$ are Lorentz vectors. In fact, 
we have the relation $\gamma^{0}\left(i \gamma_{5} \varepsilon^{\bar{V} \bar{X} \bar{Y} \bar{Z}}\right) \gamma^{0}=i \gamma_{5} \varepsilon^{V X Y Z}$. However, the pseudoscalar term $i \gamma_{5}$ is not allowed due to the relation $\gamma^{0}\left(i \gamma_{5}\right) \gamma^{0}=-i \gamma_{5}$. In the same way, the pseudoscalar term $\varepsilon^{V X Y Z}$ is not allowed. The term $\varepsilon^{A X Y Z}$ with the axial vector $A^{\mu}$ exists because of $\gamma^{0}\left(\varepsilon^{(-\bar{A}) \bar{X} \bar{Y} \bar{Z}}\right) \gamma^{0}=\varepsilon^{A X Y Z}$. The term $\sigma^{X Y}=\sigma^{\mu \nu} X_{\mu} Y_{\nu}$ is allowed under the parity invariance because of $\gamma^{0} \sigma^{\bar{X}} \bar{Y} \gamma^{0}=\sigma^{X Y}$, so that various $\sigma^{\mu \nu}$ terms exist in Eq. (20). These discussions are summarized as the properties under the parity transformation,

P-even: 1, $V, \gamma_{5} A, i \gamma_{5} V \cdot A, i \gamma_{5} \varepsilon^{V X Y Z}, \gamma_{5} \gamma_{\mu} \varepsilon^{\mu X Y Z}$, $\gamma_{\mu} \varepsilon^{\mu X Y Z}, \varepsilon^{A X Y Z}, \sigma^{X Y}, i \gamma_{5} \sigma^{A X}, \cdots$,

P-odd: $i \gamma_{5}, A, \varepsilon^{V X Y Z}, \gamma_{\mu} \varepsilon^{\mu X Y Z}, \sigma^{A X}, i \gamma_{5} \sigma^{X Y}, \ldots$

Since the parity invariance is imposed in the correlation function, the parity-odd terms do not appear in Eq. (20).

\section{Time reversal}

The time-reversal property is given in Table $\mathrm{I}$ as $\Phi^{*}(V, A, T)=\mathcal{T} \Phi(\bar{V}, \bar{A}, \bar{T}) \mathcal{T}^{-1} \quad$ where $\quad \mathcal{T}=-i \gamma_{5} C=$ $i \gamma^{1} \gamma^{3}=\mathcal{T}^{\dagger}=\mathcal{T}^{-1}=-\mathcal{T}^{*}[50]$. Because of the $\gamma$-matrix relation $\mathcal{T} \gamma^{\mu} \mathcal{T}^{-1}=\gamma^{\mu T}=\bar{\gamma}^{\mu *}$, the term $\bigvee=V_{\mu} \gamma^{\mu}$ satisfies the time-reversal relation $\mathcal{T} \bar{V} \mathcal{T}^{-1}=V^{*}$, so that it is called T-even term. In the same way, the scalar term $(\mathbf{1}$ without a $\gamma$ matrix) and the other ones $\left(\gamma_{5} A, \gamma_{5} V, i \gamma_{5} \varepsilon^{V X Y Z}, i \gamma_{5} \sigma^{A X}\right)$ satisfy the time-reversal-invariance relation, and they are T-even terms. We may note that the imaginary $i$ exists as $i \gamma_{5}$, whereas it does not exist in $\gamma_{5} \gamma^{\mu}$, because of the Hermiticity requirement $\Phi^{\dagger}=\gamma^{0} \Phi \gamma^{0}$.

However, the time-reversal relation is not satisfied for the terms with $\sigma^{X Y}, \varepsilon^{V X Y Z}$, and the others. For example, since the tensor $\sigma^{\mu \nu}$ has the property $\mathcal{T} \sigma^{\mu \nu} \mathcal{T}^{-1}=-\left(\bar{\sigma}^{\mu \nu}\right)^{*}$ under the time reversal, the term $\sigma^{X Y}$ has the relation $\mathcal{T} \sigma^{\bar{X}}{ }^{\bar{Y}} \mathcal{T}^{-1}=$ $-\left(\sigma^{X Y}\right)^{*}$ with the negative sign. This relation is same for the $i \gamma_{5} \sigma^{X Y}$ term. Therefore, they are called T-odd terms due to the negative sign. They are summarized as follows:

$$
\begin{aligned}
T \text {-even: } & \mathbf{1}, Y, \gamma_{5} A, i \gamma_{5} \varepsilon^{V X Y Z}, i \gamma_{5} \sigma^{A X}, \ldots, \\
T \text {-odd: } & i \gamma_{5}, \sigma^{X Y}, i \gamma_{5} \sigma^{X Y}, i \gamma_{5} V \cdot A, \varepsilon^{V X Y Z}, \\
& \gamma_{\mu} \varepsilon^{\mu X Y Z}, \gamma_{5} \gamma_{\mu} \varepsilon^{\mu X Y Z}, \ldots
\end{aligned}
$$

Among them, the terms $i \gamma_{5}, i \gamma_{5} \sigma^{X Y}, \varepsilon^{V X Y Z}$, and $\gamma_{\mu} \varepsilon^{\mu X Y Z}$ are ruled out by the parity invariance, so that they do not appear in Eq. (20). From this time-reversal classification, the expansion terms of Eq. (20) have the T-even and T-odd properties as

$$
\begin{array}{r}
T \text {-even terms: } A_{13-15}, A_{17}, B_{21-32}, \\
\text { T-odd terms: } A_{16}, A_{18-20}, B_{33-52} .
\end{array}
$$

Just in case, we also list the time-reversal properties in the unpolarized and vector polarization cases in Ref. [34] as

$$
\begin{aligned}
& \text { T-even terms: } A_{1-3}, A_{6-11}, B_{1}, B_{11-20}, \\
& \text { T-odd terms: } A_{4-5}, A_{12}, B_{2-10} .
\end{aligned}
$$

\section{Chirality}

The TMDs and PDFs are also classified by the chiral property. Structure functions of a hadron are given by the imaginary part of forward scattering amplitudes by the optical theorem, so that the TMDs and PDFs are expressed by parton-hadron forward scattering amplitudes in Fig. 2. The quark transversity distribution $h_{1}$ (or denoted as $\Delta_{T} q$ ) is associated with the quark spin-flip $\left(\lambda_{i}=+, \lambda_{f}=-\right)$ amplitude, so that it is called a chiral-odd distribution. This distribution is defined by the matrix element with the $\gamma$ matrix term, $i \gamma_{5} \sigma^{\mu \nu}$, as shown in Eq. (12) of Ref. [26]. At high energies, the helicity is conserved for the vector $\left(\gamma^{\mu}\right)$ and axial-vector $\left(\gamma_{5} \gamma^{\mu}\right)$ interactions. We define the righthanded and left-handed fermion states as $\psi_{R}=1 / 2\left(1-\gamma_{5}\right) \psi$ and $\psi_{L}=1 / 2\left(1+\gamma_{5}\right) \psi$, which correspond to the helicity +1 and -1 states, respectively, at high energies where the fermion masses are neglected. For example, the relation $\bar{\psi} \gamma^{\mu} \psi=\bar{\psi}_{L} \gamma^{\mu} \psi_{L}+\bar{\psi}_{R} \gamma^{\mu} \psi_{R}$ is satisfied due to the anticommutation relation $\left\{\gamma_{5}, \gamma^{\mu}\right\}=0$ and there is no cross term like $\bar{\psi}_{L} \gamma^{\mu} \psi_{R}$. This relation is also the same for the axial vector current $\gamma_{5} \gamma^{\mu}$. These facts suggest that the quark helicities should be conserved in high-energy strong, electromagnetic, and weak interactions. However, the situation is different in terms with even number of $\gamma$ matrices. The helicity is not conserved for scalar (1), axial $\left(\gamma_{5}\right)$, tensor $\sigma^{\mu \nu}$, and axialtensor $\left(i \gamma_{5} \sigma^{\mu \nu}\right)$ terms. For example, the relation becomes $\bar{\psi} 1 \psi=\bar{\psi}_{L} \psi_{R}+\bar{\psi}_{R} \psi_{L}$. Therefore, the chiral-even and odd $\gamma$ matrices are classified as

$$
\begin{aligned}
\chi \text {-even: } & \gamma^{\mu}, \gamma_{5} \gamma^{\mu}, \\
\chi \text {-odd: } & \mathbf{1}, i \gamma_{5}, \sigma^{\mu \nu}, i \gamma_{5} \sigma^{\mu \nu} .
\end{aligned}
$$

Using this classification on the chiral property, we obtain the chiral-even and chiral-odd terms of Eq. (20) as

$$
\begin{aligned}
& \chi \text {-even terms: } A_{14-15}, A_{17}, A_{20}, B_{25-40}, \\
& \chi \text {-odd terms: } A_{13}, A_{16}, A_{18-19}, A_{21-24}, B_{41-52} .
\end{aligned}
$$

The chiral properties in the unpolarized and vector polarization cases in Ref. [34] are also listed as

$\chi$-even terms: $A_{2-3}, A_{6-8}, A_{12}, B_{1}, B_{4}, B_{7-14}$,

$\chi$-odd terms: $A_{1}, A_{4-5}, A_{9-11}, B_{2-3}, B_{5-6}, B_{15-20}$.

\section{Twist of the TMDs}

Let us take the frame where the hadron's longitudinal momentum is much larger than the hadron mass, namely, $P^{+} \gg M$, by taking the hadron momentum direction as the 
third axis as given in Eq. (21), and then consider the charged-lepton deep inelastic scattering from the hadron. This frame could correspond to the center-of-momentum frame between the virtual photon emitted from the lepton and the hadron. Then, $P^{+}$is related to the scale $Q^{2}$ by the relation $P^{+} \simeq \sqrt{Q^{2} /(2 x(1+x))} \sim O(Q)$ by neglecting the hadron mass.

In the operator-product expansion, the structure functions are classified by the twist, which is the operator mass dimension minus the operator spin [57]. This twist controls the scaling behavior of the structure functions as $Q^{2}$ becomes larger. The leading-twist is two and the leading-twist structure functions or the TMDs in this work have scaling behavior with the order of $O(1)$, and the twist-3 and 4 ones are $O(1 / Q)$ and $O\left(1 / Q^{2}\right)$, respectively. Because of $P^{+} \sim O(Q)$, the leading twist-2 TMDs are defined in the TMD correlation functions as the terms of $O(1)$ as shown in Sec. III D. On the other hand, the twist-3 and twist-4 TMDs are given as the terms of $O\left(1 / P^{+}\right)$and $O\left(1 /\left(P^{+}\right)^{2}\right)$ as shown in Secs. III E and III F.

\section{Guideline for assigning TMD notations}

We follow the TMD notations of Refs. [34,40] as much as possible; however, there are new TMDs which need to be defined in this work. The twist-2 TMDs were already named for the tensor-polarized spin-1 hadron in Ref. [40], and the same notations are used in twist 2. However, all the twist-3 and twist- 4 TMDs are new ones for the tensor-polarized spin-1 hadron, so that new names should be assigned. In the twist-3 part, our notations are given in the similar spirit to the twist-3 TMDs of the spin-1/2 nucleon in Ref. [34]. In twist 4 , the TMD names are given by replacing all the twist- 2 subscripts 1 (such as in $\left.f_{1 L L}\right)$ by twist- 4 ones $3\left(f_{3 L L}\right)$. The general guideline is the following.

(1) The TMD function names $f, g$, and $h$ are assigned to the unpolarized, longitudinal, and transverse quark polarizations by taking traces of Eq. (24) with $\gamma^{+}$, $\gamma^{+} \gamma_{5}$, and $i \sigma^{i+} \gamma_{5}\left(\right.$ or $\left.\sigma^{i+}\right)$, respectively, in the twist-2 case. The quark operators $\bar{\psi} \gamma^{+} \psi, \bar{\psi} \gamma^{+} \gamma_{5} \psi$, and $\bar{\psi} i \sigma^{i+} \gamma_{5} \psi$ are related to the unpolarized (U), longitudinally polarized, and transvsere polarization (T) of a quark in the twist-2 case as given in Ref. [26]. However, the twist-3 and twist-4 TMDs indicate three and four parton correlations, so that they are not related to these quark distributions. Therefore, the distributions $f, g$, and $h$ are assigned depending on the operator forms as shown in Tables IV and VI.

(2) The subscript 1 in the TMDs, such as $f_{1 L L}$, is assigned for the twist-2 TMDs. The subscript 3 is used for the twist-4 TMDs like $f_{3 L L}$; however, the subscript 2 is not conventionally used for expressing the twist-3 TMDs.

(3) The subscripts $L L, S L$, and $T T$ are given if TMDs appear with the spin parameters $S_{L L}, S_{L T}$, and $S_{T T}$, respectively, in the traces of the TMD correlation functions of Eq. (24). For example, $f_{1 L L}, f_{1 L T}$, and $f_{1 L T}$ are defined in Eq. (33) in this way.

(4) The superscript $\perp\left(F^{\perp}\right)$ is given if a TMD exists with the partonic transverse momentum $k_{T}^{i}(i=1,2)$. In addition, the superscript ' $\left(F^{\prime}\right)$ is assigned if two similar polarization and momentum factors exist within the same TMD correlation function $\Phi^{[\Gamma]}$. If both $F^{\perp}$ and $F^{\prime}$ exist, the superscript $\perp$ is assigned for the term with the partonic transverse-momentum $\left(k_{T}\right)$ term of the order of $\left(k_{T}\right)^{2}$ or $\left(k_{T}\right)^{3}$ in traces of TMD correlation functions. An example is $h_{1 L T}^{\perp}$ in Eq. (33). However, although the corresponding $F^{\prime}$ does not exist, the $F^{\perp}$ could be used even in the order of $\left(k_{T}\right)^{1}$, and its example is $h_{1 L L}^{\perp}$. The TMDs with ' are assigned in the leading order of $k_{T}$, namely, $O\left(\left(k_{T}\right)^{0}\right)$ or $O\left(\left(k_{T}\right)^{1}\right)$. An example is $h_{1 L T}^{\prime}$ in Eq. (33). In general, new TMDs $F$ are defined from the TMDs $F^{\perp}$ and $F^{\prime}$ by the relation of Eq. (36), so that the TMD lists are shown by the two-independent TMDs $F$ and $F^{\perp}$ without $F^{\prime}$ in Tables II, IV, and VI. If both $F$ and $F^{\perp}$ exist, the meaning of $F$ and $F^{\perp}$ is more clearly shown in Eq. (35). The function $F^{\perp}$ [e.g., $h_{1 L T}^{\perp}$ in Eq. (35)] is given as the term with a kinematical factor which vanishes by the $\vec{k}_{T}$ integration. The other function $F$ (e.g., $h_{1 L T}$ ) is assigned for the remaining part.

(5) There are exceptions for the above assignment of $\perp$. The superscript $\perp$ is not written conventionally for $f_{1 L T}, f_{1 T T}, g_{1 L T}, g_{1 T T}$, (also the twist-4 distributions $f_{3 L T}, f_{3 T T}, g_{3 L T}$, and $g_{3 T T}$ ), although they accompany $k_{T}$ factors in the correlation functions.

(6) The superscript ' is not written if similar functions exist in separate correlation functions $\Phi^{\left[\Gamma_{1}\right]}$ and $\Phi^{\left[\Gamma_{2}\right]}$. In the traces for the twist-3 TMD correlation functions $\Phi^{\left[\sigma^{-+}\right]}$and $\Phi^{\left[\sigma^{i j}\right]}$ in Eq. (46), the $k_{T}$ dependence is the same order $O\left(\left(k_{T}\right)^{1}\right)$ for $h_{L T}^{\perp}$, so one may assign $h_{L T}^{\perp(1)}$ and $h_{L T}^{\perp(2)}$. Similar expressions appeared in the twist-3 part of the nucleon, and they were already named as $h_{T}$ and $h_{T}^{\perp}$ in Ref. [34]. Following such a convention, we write them as $h_{L T}$ and $h_{L T}^{\perp}$ in Eq. (46). In this equation, $h_{T T}$ and $h_{T T}^{\perp}$ are also written in the same manner in Eq. (46), although they have the same dependence of $O\left(\left(k_{T}\right)^{0}\right)$. In the same way, $e_{L T}, e_{L T}^{\perp}, e_{T T}$, and $e_{T T}^{\perp}$ are assigned in $\Phi^{[1]}$ and $\Phi^{\left[\gamma_{5}\right]}$.

\section{Twist-2 TMDs for a tensor-polarized spin-1 hadron}

The leading twist TMDs for a tensor-polarized spin-1 hadron are defined by taking $\Gamma=\gamma^{+}, \gamma^{+} \gamma_{5}$, and $\sigma^{i+}$ in Eq. (24), and we obtain 


$$
\begin{aligned}
\Phi^{\left[\gamma^{+}\right]}\left(x, k_{T}, T\right)= & f_{1 L L}\left(x, k_{T}^{2}\right) S_{L L}-f_{1 L T}\left(x, k_{T}^{2}\right) \frac{S_{L T} \cdot k_{T}}{M} \\
& +f_{1 T T}\left(x, k_{T}^{2}\right) \frac{k_{T} \cdot S_{T T} \cdot k_{T}}{M^{2}} \\
\Phi^{\left[\gamma^{+} \gamma_{5}\right]}\left(x, k_{T}, T\right)= & g_{1 L T}\left(x, k_{T}^{2}\right) \frac{S_{L T \mu} \varepsilon_{T}^{\mu \nu} k_{T \nu}}{M} \\
& +g_{1 T T}\left(x, k_{T}^{2}\right) \frac{S_{T T \mu \rho} k_{T}^{\rho} \varepsilon_{T}^{\mu \nu} k_{T \nu}}{M^{2}} \\
\Phi^{\left[\sigma^{i+}\right]}\left(x, k_{T}, T\right)= & h_{1 L L}^{\perp}\left(x, k_{T}^{2}\right) \frac{S_{L L} k_{T}^{i}}{M}+h_{1 L T}^{\prime}\left(x, k_{T}^{2}\right) S_{L T}^{i} \\
& -h_{1 L T}^{\perp}\left(x, k_{T}^{2}\right) \frac{k_{T}^{i} S_{L T} \cdot k_{T}}{M^{2}} \\
& -h_{1 T T}^{\prime}\left(x, k_{T}^{2}\right) \frac{S_{T T}^{i j} k_{T j}}{M} \\
& +h_{1 T T}^{\perp}\left(x, k_{T}^{2}\right) \frac{k_{T} \cdot S_{T T} \cdot k_{T}}{M^{2}} \frac{k_{T}^{i}}{M}
\end{aligned}
$$

where $i$ and $j$ indicate the transverse indices $i=1,2$ $(j=1,2), \varepsilon_{T}^{\mu \nu}=\varepsilon^{\mu \nu \alpha \beta} \bar{n}_{\alpha} n_{\beta}$ is used with the convention $\varepsilon^{0123}=1$, and $S_{L T} \cdot k_{T}$ and $k_{T} \cdot S_{T T} \cdot k_{T}$ are defined by $S_{L T} \cdot k_{T}=S_{L T}^{i} k_{T i}=-S_{L T}^{i} k_{T}^{i}$ and $k_{T} \cdot S_{T T} \cdot k_{T}=k_{T i} S_{T T}^{i j} k_{T j}$. Here, we follow the notations of Ref. [40] for the TMD expressions in twist 2. In Ref. [40], the trace with $i \sigma^{i+} \gamma_{5}$ was taken instead of $\sigma^{i+}$; however, both formalisms are equivalent by considering the relation $i \sigma^{\mu \nu} \gamma_{5}=$ $-\varepsilon^{\mu \nu \alpha \beta} \sigma_{\alpha \beta} / 2$ [51]. Therefore, if $\Phi^{\left[i \sigma^{i+} \gamma_{5}\right]}$ is calculated, the same equation is obtained by the replacements $X^{i} \rightarrow \varepsilon_{T}^{i j} X_{j}$ with $X^{i}=k_{T}^{i}, S_{L T}^{i}$, and $S_{T T}^{i j} k_{T j}$ in $\Phi^{\left[\sigma^{i+}\right]}$ of Eq. (33). There are ten TMDs in the leading-twist level, as already found in Ref. [40]. However, their relations to the expansion coefficients are modified due to the existence of the new terms $B_{21-52}$ associated with the tensor structure and the lightlike vector $n$, as we find in Eqs. (37)-(39).

The two TMDs $h_{1 L T}^{\prime}\left(h_{1 T T}^{\prime}\right)$ and $h_{1 L T}^{\perp}\left(h_{1 T T}^{\perp}\right)$ are similar notations. Because of the relation

$$
k_{T}^{i} k_{T} \cdot S_{L T}=\varepsilon_{T}^{i j} k_{T j} k_{T l} \varepsilon_{T}^{l m} S_{L T m}+k_{T}^{2} S_{L T}^{i}
$$

the other functions $h_{1 L T}$ and $h_{1 T T}$ could be defined instead

\begin{tabular}{|c|c|c|c|c|c|c|}
\hline \multirow[b]{3}{*}{ Hadron } & \multicolumn{6}{|c|}{ Quark } \\
\hline & \multicolumn{2}{|c|}{$\mathrm{U}\left(\gamma^{+}\right)$} & \multicolumn{2}{|c|}{$\mathrm{L}\left(\gamma^{+} \gamma_{5}\right)$} & \multicolumn{2}{|c|}{$\mathrm{T}\left(i \sigma^{i+} \gamma_{5} / \sigma^{i+}\right)$} \\
\hline & T-even & T-odd & T-even & T-odd & T-even & T-odd \\
\hline$\overline{\mathrm{U}}$ & $f_{1}$ & & & & & {$\left[h_{1}^{\perp}\right]$} \\
\hline $\mathrm{L}$ & & & $g_{1 L}$ & & {$\left[h_{1 L}^{\perp}\right]$} & \\
\hline $\mathrm{T}$ & & $f_{1 T}^{\perp}$ & $g_{1 T}$ & & {$\left[h_{1}\right],\left[h_{1 T}^{\perp}\right]$} & \\
\hline LL & $f_{1 L L}$ & & & & & {$\left[h_{1 L L}^{\perp}\right]$} \\
\hline $\mathrm{LT}$ & $f_{1 L T}$ & & & $g_{1 L T}$ & & {$\left[h_{1 L T}\right],\left[h_{1 L T}^{\perp}\right.$} \\
\hline TT & $f_{1 T T}$ & & & $g_{1 T T}$ & & {$\left[h_{1 T T}\right],\left[h_{1 T T}^{\perp}\right.$} \\
\hline
\end{tabular}
of $h_{1 L T}^{\prime}$ and $h_{1 T T}^{\prime}$. In fact, the correlation function $\Phi^{\left[\sigma^{i+}\right]}$ in Eq. (33) is rewritten as

$$
\begin{aligned}
\Phi^{\left[\sigma^{i+}\right]}= & h_{1 L L}^{\perp} \frac{S_{L L} k_{T}^{i}}{M} \\
& +h_{1 L T} S_{L T}^{i}+h_{1 L T}^{\perp} \frac{S_{L T}^{j} k_{T}^{i} k_{T}^{j}-S_{L T}^{i} \vec{k}_{T}^{2} / 2}{M^{2}} \\
& +h_{1 T T} \frac{S_{T T}^{i j} k_{T}^{j}}{M}+h_{1 T T}^{\perp} \frac{\left(S_{T T}^{l j} k_{T}^{i} k_{T}^{l}-S_{T T}^{i j} \vec{k}_{T}^{2} / 2\right) k_{T}^{j}}{M^{3}} .
\end{aligned}
$$

TABLE II. List of twist-2 quark TMDs for a spin-1 hadron in terms of the quark and hadron polarizations. The square brackets [ ] indicate chiral-odd distributions and the others are chiral-even ones.

Here, we define the new functions without ' and $\perp$ as

$$
F\left(x, k_{T}^{2}\right) \equiv F^{\prime}\left(x, k_{T}^{2}\right)-\frac{k_{T}^{2}}{2 M^{2}} F^{\perp}\left(x, k_{T}^{2}\right),
$$

where $F=h_{1 L T}$ and $h_{1 T T}$ in the twist 2 and $k_{T}^{2}=-\vec{k}_{T}^{2}$, as this relation was written for the unpolarized TMD $\left(f, f^{\prime}\right.$, and $f^{\perp}$ ) in Ref. [52]. We note in Eq. (36) that the $h_{1 L T}^{\perp}$ and $h_{1 T T}^{\perp}$ terms vanish by the $\vec{k}_{T}$ integration. It leads to the sum rule for $f_{1 L T}$ in Eq. (57). Therefore, two of these three functions $h_{1 L T}, h_{1 L T}^{\prime}$, and $h_{1 L T}^{\perp}$ (also $h_{1 T T}, h_{1 T T}^{\prime}$, and $h_{1 T T}^{\perp}$ ) are independent, so that one could choose two of them depending on one's preference in defining the TMDs in Eq. (33). Similar relations appear in twist-3 and twist- 4 cases, so that we use Eq. (36) as the general relation for the TMD $F$ in terms of $F^{\prime}$ and $F^{\perp}$.

Calculating traces in Eq. (24) with the new correlation function of Eq. (20), we express the twist-2 TMDs in Eq. (33) in terms of the coefficients $A_{i}$ and $B_{i}$. First, the unpolarized quark TMDs in $\Phi^{\left[\gamma^{+}\right]}$are given as

$$
\begin{aligned}
f_{1 L L}\left(x, k_{T}^{2}\right)= & \frac{P^{+}}{3} \int d k^{-}\left[\left(A_{14}+x A_{15}\right) \tau_{x}\right. \\
& +2\left(A_{17}+B_{28}+x B_{30}\right)(\sigma-2 x) \\
& \left.+4\left(B_{29}+x B_{31}+B_{32}\right)\right], \\
f_{1 L T}\left(x, k_{T}^{2}\right)= & -P^{+} \int d k^{-}\left[\left(A_{14}+x A_{15}\right)(\sigma-2 x)\right. \\
& \left.+A_{17}+B_{28}+x B_{30}\right], \\
f_{1 T T}\left(x, k_{T}^{2}\right)= & P^{+} \int d k^{-}\left(A_{14}+x A_{15}\right),
\end{aligned}
$$

where $\tau_{x}$ is defined by $\tau_{x}=\sigma^{2}-6 x \sigma+2 \tau+6 x^{2}$. The terms of $A_{14}, A_{15}, \ldots$, and $B_{32}$ are time-reversal even (T-even) and chiral even ( $\chi$-even) terms as given in Eqs. (28) and (31), so that these TMDs are T-even and $\chi$-even ones as listed in Table II. In this table, the TMDs of the unpolarized (U), longitudinally polarized (L), and 
transversely polarized $(\mathrm{T})$ hadron are also listed for showing the complete set of the TMDs of the spin-1 hadron in addition to the tensor polarizations $L L, L T$, and $T T$. As explained after Eq. (18), the notation LL indicates the tensor polarization along the longitudinal axis, and the notations LT and TT are for the polarization differences shown in the Appendix of Ref. [40].

Next, the longitudinally polarized quark TMDs in $\Phi^{\left[\gamma^{+} \gamma_{5}\right]}$ are given as

$$
\begin{aligned}
& g_{1 L T}\left(x, k_{T}^{2}\right)=-\frac{P^{+}}{2} \int d k^{-}\left[A_{20}(\sigma-2 x)+2 B_{33}\right], \\
& g_{1 T T}\left(x, k_{T}^{2}\right)=-P^{+} \int d k^{-} A_{20} .
\end{aligned}
$$

Because of the time-reversal and chiral properties of the $A_{20}$ and $B_{33}$ terms, these TMDs are T-odd and $\chi$-even distributions as listed in Table II. Third, the transversely polarized quark TMDs in $\Phi^{\left[\sigma^{i+}\right]}$ are given as

$$
\begin{aligned}
h_{1 L L}^{\perp}\left(x, k_{T}^{2}\right)= & -\frac{P^{+}}{3} \int d k^{-}\left[A_{16} \tau_{x}+2 A_{19}(\sigma-3 x)\right. \\
& \left.+2 B_{41}(\sigma-2 x)-2\left(A_{18}-2 B_{42}-2 B_{52}\right)\right], \\
h_{1 L T}^{\prime}\left(x, k_{T}^{2}\right)= & \frac{P^{+}}{2} \int d k^{-}\left[\left(A_{18}+x A_{19}\right)(\sigma-2 x)\right. \\
& \left.+2\left(B_{51}+x B_{52}\right)\right], \\
h_{1 L T}^{\perp}\left(x, k_{T}^{2}\right)= & P^{+} \int d k^{-}\left[A_{16}(\sigma-2 x)+A_{19}+B_{41}\right], \\
h_{1 T T}^{\prime}\left(x, k_{T}^{2}\right)= & -P^{+} \int d k^{-}\left(A_{18}+x A_{19}\right), \\
h_{1 T T}^{\perp}\left(x, k_{T}^{2}\right)= & -P^{+} \int d k^{-} A_{16} .
\end{aligned}
$$

These TMDs are T-odd and $\chi$-odd distributions as shown in Table II. Here, the TMDs $h_{1 L T}$ and $h_{1 T T}$ are listed instead of $h_{1 L T}^{\prime}$ and $h_{1 T T}^{\prime}$ due to the relation of Eq. (36). In comparison with previous works [21,40], the new terms exist in association with the light cone vector $n$ and the tensor polarizations, namely, the new coefficients $B_{21-52}$. Therefore, the expressions of $f_{1 L L}, f_{1 L T}, g_{1 L T}, h_{1 L L}^{\perp}, h_{1 L T}^{\prime}$, and $h_{1 L T}^{\perp}$ are modified from previous ones due to the existence of the new terms, $B_{28-33}, B_{41,42}$, and $B_{51,52}$.

The collinear PDFs are obtained from the TMDs by integrating them over the partonic transverse momentum as

$$
f(x)=\int d^{2} k_{T} f\left(x, k_{T}^{2}\right) .
$$

Since the time-reversal invariance is satisfied in QCD, the

\begin{tabular}{|c|c|c|c|c|c|c|}
\hline \multirow{3}{*}{ Hadron } & \multicolumn{6}{|c|}{ Quark } \\
\hline & \multicolumn{2}{|c|}{$\mathrm{U}\left(\gamma^{+}\right)$} & \multicolumn{2}{|c|}{$\mathrm{L}\left(\gamma^{+} \gamma_{5}\right)$} & \multicolumn{2}{|c|}{$\mathrm{T}\left(\mathrm{i} \sigma^{\mathrm{i}+} \gamma_{5} / \sigma^{\mathrm{i}+}\right)$} \\
\hline & T-even & T-odd & T-even & T-odd & T-even & T-odd \\
\hline $\mathrm{U}$ & $f_{1}$ & & & & & \\
\hline $\mathrm{L}$ & & & $g_{1 L}\left(g_{1}\right)$ & & & \\
\hline $\mathrm{T}$ & & & & & {$\left[h_{1}\right]$} & \\
\hline $\begin{array}{l}\text { LL } \\
\text { LT }\end{array}$ & $f_{1 L L}\left(b_{1}\right)$ & & & & & $* 1$ \\
\hline TT & & & & & & \\
\hline
\end{tabular}
T-odd collinear PDFs should vanish $[34,38,58]$,

$$
f(x)_{\mathrm{T} \text {-odd }}=0,
$$

TABLE III. List of twist-2 quark collinear PDFs for a spin-1 hadron in terms of the quark and hadron polarizations. The square bracket [] indicates a chiral-odd distribution and the others are chiral-even ones. The function $g_{1 L}, h_{1}$, and $f_{1 L L}$ are often denoted as $g_{1}$ or $\Delta q,-\Delta_{T} q$, and $-(2 / 3) b_{1}$ or $-(2 / 3) \delta_{T} q$ [26]. ${ }^{\text {a }}$ The asterisk $* 1$ is explained in the main text.

${ }^{\mathrm{a}}$ The functions $g_{1 L}$ and $f_{1 L L}$ are sometimes listed by $g_{1}$ and $b_{1}$ as for the abbreviated notations of $g_{1, q}$ and $b_{1, q}$. These $g_{1}$ and $b_{1}$, and also $h_{1}$, should not be confused with the structure function themselves including charge-squared factors and coefficient functions.

although the T-odd TMDs exist in Table II due to the gauge link including the transverse direction. Therefore, the only remaining PDFs are $f_{1}, g_{1 L}$ (or often denoted as $g_{1}$ or $\Delta q$ ), $h_{1}\left(\Delta_{T} q\right)$, and $f_{1 L L}\left(b_{1}\right.$ or $\left.\delta_{T} q\right)$ as shown in Table III. The $h_{1}(x)$ is defined from the TMDs $h_{1 T}$ and $h_{1 T}^{\perp}$ as $h_{1}(x)=$ $\int d^{2} k_{T}\left[h_{1 T}\left(x, k_{T}^{2}\right)-k_{T}^{2} /\left(2 M^{2}\right) h_{1 T}^{\perp}\left(x, k_{T}^{2}\right)\right]$ [40]. The number of twist-2 quark distributions is 4 in Table 3 of Ref. [57] and this number 4 agrees with the existence of four distributions $f_{1}, g_{1}, h_{1}$, and $f_{1 L L}\left(b_{1}\right)$ in Table III. The only tensor-polarized twist-2 PDF is $f_{1 L L}\left(b_{1}\right.$ or $\left.\delta_{T} q\right)$ which is associated with the spin-1 nature of the hadron. The asterisk $(* 1)$ in Table III indicates the following. Because of the time-reversal invariance, the collinear PDF $h_{1 L T}(x)$ vanish as shown in Eq. (41). However, since the timereversal invariance cannot be imposed in the fragmentation functions, we should note that the corresponding fragmentation function $H_{1 L T}(z)$ [25], as indicated by the replacements of Eq. (56), should exist as a collinear fragmentation function.

In addition to the T-odd functions, some of T-even functions disappear after the $\vec{k}_{T}$ integration. For example, if the correlation function $\Phi^{\left[\gamma^{+}\right]}\left(x, k_{T}, T\right)$ in Eq. (33) is integrated to obtain the collinear correlation function

$$
\Phi^{[\Gamma]}(x, T)=\int d^{2} k_{T} \Phi^{[\Gamma]}\left(x, k_{T}, T\right),
$$

where $\Gamma=\gamma^{+}$here, the second term vanishes, and the third term also vanishes due to $S_{T T}^{x x}=-S_{T T}^{y y}$ [26], so that the collinear PDFs $f_{1 L T}(x)$ and $f_{1 T T}(x)$ do not exist. In the same way, the functions $g_{1 L T}, g_{1 T T}$, and $h_{1 L}$ do not exist in Table III. 


\section{E. Twist-3 TMDs for a tensor-polarized spin-1 hadron}

For the spin-1/2 nucleon, the twist-3 TMDs are listed in the quark correlation function including new terms with the light cone vector $n$ in Ref. [34]. Here, we list all the possible twist-3 TMDs in the quark correlation function for a tensor-polarized spin- 1 hadron, so that all the following terms are new ones we found in this work. The twist-3 TMDs with the $1 / P^{+}$dependence are found by considering $\Phi^{\left[\gamma^{i}\right]}, \Phi^{[1]}, \Phi^{\left[\gamma_{5}\right]} \Phi^{\left[\gamma^{i} \gamma_{5}\right]} \Phi^{\left[\sigma^{i j}\right]}$, and $\Phi^{\left[\sigma^{-+}\right]}$. First, the TMDs with the function name $f$ are defined for the quark operator type $\bar{\psi} \gamma^{i} \psi$ as

$$
\begin{aligned}
\Phi^{\left[\gamma^{i}\right]}\left(x, k_{T}, T\right)= & \frac{M}{P^{+}}\left[f_{L L}^{\perp}\left(x, k_{T}^{2}\right) S_{L L} \frac{k_{T}^{i}}{M}+f_{L T}^{\prime}\left(x, k_{T}^{2}\right) S_{L T}^{i}-f_{L T}^{\perp}\left(x, k_{T}^{2}\right) \frac{k_{T}^{i} S_{L T} \cdot k_{T}}{M^{2}}-f_{T T}^{\prime}\left(x, k_{T}^{2}\right) \frac{S_{T T}^{i j} k_{T j}}{M}\right. \\
& \left.+f_{T T}^{\perp}\left(x, k_{T}^{2}\right) \frac{k_{T} \cdot S_{T T} \cdot k_{T}}{M^{2}} \frac{k_{T}^{i}}{M}\right] .
\end{aligned}
$$

These $f$-type TMDs have T-even and $\chi$-even properties. The distributions $f_{L T}, f_{L T}^{\prime}$, and $f_{L T}^{\perp}\left(f_{T T}, f_{T T}^{\prime}\right.$, and $\left.f_{T T}^{\perp}\right)$ are related by the relation of Eq. (36). The TMDs with the name $e$ are assigned for the currents associated with $\bar{\psi} 1 \psi$ and $\bar{\psi} i \gamma_{5} \psi$ as

$$
\begin{aligned}
\Phi^{[1]}\left(x, k_{T}, T\right) & =\frac{M}{P^{+}}\left[e_{L L}\left(x, k_{T}^{2}\right) S_{L L}-e_{L T}^{\perp}\left(x, k_{T}^{2}\right) \frac{S_{L T} \cdot k_{T}}{M}+e_{T T}^{\perp}\left(x, k_{T}^{2}\right) \frac{k_{T} \cdot S_{T T} \cdot k_{T}}{M^{2}}\right], \\
\Phi^{\left[\gamma_{5}\right]} & =\frac{M}{P^{+}}\left[e_{L T}\left(x, k_{T}^{2}\right) \frac{S_{L T \mu} \varepsilon_{T}^{\mu \nu} k_{T \nu}}{M}-e_{T T}\left(x, k_{T}^{2}\right) \frac{S_{T T \mu \rho} k_{T}^{\rho} \varepsilon_{T}^{\mu \nu} k_{T \nu}}{M^{2}}\right] .
\end{aligned}
$$

These $e$-type TMDs have T-even and $\chi$-odd properties. The distributions $e_{L T}$ and $e_{T L}^{\perp}$ are given with the same factors of $O\left(\left(k_{T}\right)^{1}\right)$; however, we assigned $e_{L T}^{\perp}$ for the first one and $e_{L T}$ for the second as explained in the guideline 6 of Sec. III C. The distributions $e_{T T}$ and $e_{T T}^{\perp}$ are also named in the same way. Next, the $g$-type TMDs with T-odd and $\chi$-even properties are defined for the current $\bar{\psi} \gamma^{i} \gamma_{5} \psi$ as

$$
\begin{aligned}
\Phi^{\left[\gamma^{i} \gamma_{5}\right]}\left(x, k_{T}, T\right)= & \frac{M}{P^{+}}\left[-g_{L L}^{\perp}\left(x, k_{T}^{2}\right) S_{L L} \frac{\varepsilon_{T}^{i j} k_{T j}}{M}-g_{L T}^{\prime}\left(x, k_{T}^{2}\right) \varepsilon_{T}^{i j} S_{L T j}+g_{L T}^{\perp}\left(x, k_{T}^{2}\right) \frac{\varepsilon_{T}^{i j} k_{T j} S_{L T} \cdot k_{T}}{M^{2}}\right. \\
& \left.+g_{T T}^{\prime}\left(x, k_{T}^{2}\right) \frac{\varepsilon_{T}^{i j} S_{T T j l} k_{T}^{l}}{M}-g_{T T}^{\perp}\left(x, k_{T}^{2}\right) \frac{k_{T} \cdot S_{T T} \cdot k_{T}}{M^{2}} \frac{\varepsilon_{T}^{i j} k_{T j}}{M}\right] .
\end{aligned}
$$

The distributions $g_{L T}, g_{L T}^{\prime}$, and $g_{L T}^{\perp}\left(g_{T T}, g_{T T}^{\prime}\right.$, and $\left.g_{T T}^{\perp}\right)$ are related by the relation of Eq. (36). The $h$-type TMDs with the T-odd and $\chi$-odd properties are given for the currents $\bar{\psi} \sigma^{-+} \psi$ and $\bar{\psi} \sigma^{i j} \psi$ as

$$
\begin{gathered}
\Phi^{\left[\sigma^{-+}\right]}\left(x, k_{T}, T\right)=\frac{M}{P^{+}}\left[h_{L L}\left(x, k_{T}^{2}\right) S_{L L}-h_{L T}\left(x, k_{T}^{2}\right) \frac{S_{L T} \cdot k_{T}}{M}+h_{T T}\left(x, k_{T}^{2}\right) \frac{k_{T} \cdot S_{T T} \cdot k_{T}}{M^{2}}\right], \\
\Phi^{\left[\sigma^{i j}\right]}\left(x, k_{T}, T\right)=\frac{M}{P^{+}}\left[h_{L T}^{\perp}\left(x, k_{T}^{2}\right) \frac{S_{L T}^{i} k_{T}^{j}-S_{L T}^{j} k_{T}^{i}}{M}-h_{T T}^{\perp}\left(x, k_{T}^{2}\right) \frac{S_{T T}^{i l} k_{T l} k_{T}^{j}-S_{T T}^{j l} k_{T l} k_{T}^{i}}{M^{2}}\right] .
\end{gathered}
$$

The prime marks $\left({ }^{\prime}\right)$ are not assigned for $h_{L T}$ and $h_{T T}$ because of the guideline 6 in Sec. III C.

There are twenty TMDs in the twist-3 for a tensorpolarized spin- 1 hadron. These TMDs are expressed by the expansion coefficients of the correlation function. First, we obtain the $f$-type TMDs as

$$
\begin{aligned}
f_{L L}^{\perp}\left(x, k_{T}^{2}\right)= & \frac{P^{+}}{3} \int d k^{-}\left[A_{15} \tau_{x}+2 A_{17}\right. \\
& \left.+2 B_{30}(\sigma-2 x)+4 B_{31}\right], \\
f_{L T}^{\prime}\left(x, k_{T}^{2}\right)= & \frac{P^{+}}{2} \int d k^{-}\left[A_{17}(\sigma-2 x)+2 B_{32}\right],
\end{aligned}
$$

$$
\begin{aligned}
f_{L T}^{\perp}\left(x, k_{T}^{2}\right) & =-P^{+} \int d k^{-}\left[A_{15}(\sigma-2 x)+B_{30}\right], \\
f_{T T}^{\prime}\left(x, k_{T}^{2}\right) & =-P^{+} \int d k^{-} A_{17} \\
f_{T T}^{\perp}\left(x, k_{T}^{2}\right) & =P^{+} \int d k^{-} A_{15} .
\end{aligned}
$$

The terms with $A_{15}, A_{17}, \ldots, B_{32}$ are T-even and $\chi$-even as listed in Eqs. (28) and (31), so that these TMDs are T-even and $\chi$-even properties as shown in Table IV. The e-type TMDs are expressed as 
TABLE IV. List of twist-3 quark TMDs for a spin-1 hadron in terms of the hadron polarizations and the operator forms in the correlation functions. The square brackets [] indicate chiral-odd distributions and the others are chiraleven ones. The LL, LT, and TT TMDs are new distributions found in this work.

\begin{tabular}{|c|c|c|c|c|c|c|}
\hline \multirow{3}{*}{ Hadron } & \multicolumn{6}{|c|}{ Quark } \\
\hline & \multicolumn{2}{|c|}{$\gamma^{\mathrm{i}}, 1, \mathrm{i} \gamma_{5}$} & \multicolumn{2}{|c|}{$\gamma^{+} \gamma_{5}$} & \multicolumn{2}{|c|}{$\sigma^{\mathrm{ij}}, \sigma^{-+}$} \\
\hline & T-even & T-odd & T-even & $\overline{\text { T-odd }}$ & T-even & T-odd \\
\hline $\mathrm{U}$ & $f^{\perp}[e]$ & & & $g^{\perp}$ & & {$[h]$} \\
\hline $\mathrm{L}$ & & $f_{L}^{\perp}\left[e_{L}\right]$ & $g_{L}^{\perp}$ & & {$\left[h_{L}\right]$} & \\
\hline $\mathrm{T}$ & & $f_{T}, f_{T}^{\perp}\left[e_{T}, e_{T}^{\perp}\right]$ & $g_{T}, g_{T}^{\perp}$ & & {$\left[h_{T}\right],\left[h_{T}^{\perp}\right]$} & \\
\hline LL & $f_{L L}^{\perp}\left[e_{L L}\right]$ & & & $g_{L L}^{\perp}$ & & {$\left[h_{L L}\right]$} \\
\hline LT & $f_{L T}, f_{L T}^{\perp}\left[e_{L T}, e_{L T}^{\perp}\right]$ & & & $g_{L T}, g_{L T}^{\perp}$ & & {$\left[h_{L T}\right],\left[h_{L T}^{\perp}\right]$} \\
\hline TT & $f_{T T}, f_{T T}^{\perp}\left[e_{T T}, e_{T T}^{\perp}\right]$ & & & $g_{T T}, g_{T T}^{\perp}$ & & {$\left[h_{T T}\right],\left[h_{T T}^{\perp}\right]$} \\
\hline
\end{tabular}

$e_{L L}\left(x, k_{T}^{2}\right)=\frac{P^{+}}{3} \int d k^{-}\left[A_{13} \tau_{x}+2 B_{21}(\sigma-2 x)+4 B_{22}\right]$

$e_{L T}^{\perp}\left(x, k_{T}^{2}\right)=-P^{+} \int d k^{-}\left[A_{13}(\sigma-2 x)+B_{21}\right]$,

$e_{T T}^{\perp}\left(x, k_{T}^{2}\right)=P^{+} \int d k^{-} A_{13}$,

$e_{L T}\left(x, k_{T}^{2}\right)=P^{+} \int d k^{-}\left[B_{23}\left(x-\frac{\sigma}{2}\right)-B_{24}\right]$,

$e_{T T}\left(x, k_{T}^{2}\right)=P^{+} \int d k^{-} B_{23}$.

Because of the terms $A_{13}, B_{21}, \ldots, B_{24}$, these TMDs have properties of T-even and $\chi$-odd as shown in Table IV.

Second, we obtain the $g$-type TMDs as

$$
\begin{aligned}
g_{L L}^{\perp}\left(x, k_{T}^{2}\right)= & \frac{P^{+}}{3} \int d k^{-}\left[3 A_{20}(\sigma-2 x)+4 B_{33}+2 B_{34}\right. \\
& \left.+B_{36} \tau_{x}+2 B_{37}(\sigma-2 x)+4 B_{38}\right], \\
g_{L T}^{\prime}\left(x, k_{T}^{2}\right)= & \frac{P^{+}}{4} \int d k^{-}\left[A_{20}(\sigma-2 x)^{2}\right. \\
& \left.+2\left(B_{33}+B_{34}\right)(\sigma-2 x)+4 B_{35}\right], \\
g_{L T}^{\perp}\left(x, k_{T}^{2}\right)= & -P^{+} \int d k^{-}\left[A_{20}+B_{36}(\sigma-2 x)+B_{37}\right], \\
g_{T T}^{\prime}\left(x, k_{T}^{2}\right)= & -P^{+} \int d k^{-}\left[A_{20}\left(\frac{\sigma}{2}-x\right)+B_{34}\right], \\
g_{T T}^{\perp}\left(x, k_{T}^{2}\right)= & P^{+} \int d k^{-} B_{36} .
\end{aligned}
$$

Because of the terms $A_{20}, \cdots, B_{38}$, these TMDs have properties of T-odd and $\chi$-even as shown in Table IV. Three new twist-3 TMDs $e_{L T}, e_{T T}$, and $g_{T T}^{\perp}$ are expressed purely by the new terms $B_{20-52}$.
Third, the $h$-type TMDs are expressed as

$$
\begin{aligned}
h_{L L}\left(x, k_{T}^{2}\right)= & \frac{P^{+}}{6} \int d k^{-}\left\{(\sigma-2 x)\left[A_{16} \tau_{x}+2\left(2 A_{18}+\sigma A_{19}\right)\right]\right. \\
& +2(\sigma-2 x)\left[B_{41}(\sigma-2 x)\right. \\
& \left.+2\left(B_{42}+B_{44}+x B_{47}+B_{49}\right)\right] \\
& +2\left(B_{43}+x B_{46}\right) \tau_{x} \\
& \left.+4\left(2 B_{45}+2 x B_{48}+2 B_{50}+2 B_{51}+\sigma B_{52}\right)\right\}, \\
h_{L T}\left(x, k_{T}^{2}\right)= & -\frac{P^{+}}{2} \int d k^{-}\left[A_{16}(\sigma-2 x)^{2}+2 A_{18}+\sigma A_{19}\right. \\
& +(\sigma-2 x)\left(B_{41}+2 B_{43}+2 x B_{46}\right) \\
& \left.+2\left(B_{44}+x B_{47}+B_{49}\right)\right], \\
h_{L T}^{\perp}\left(x, k_{T}^{2}\right)= & \frac{P^{+}}{2} \int d k^{-}\left[A_{19}(\sigma-2 x)+2 B_{52}\right], \\
h_{T T}\left(x, k_{T}^{2}\right)= & \frac{P^{+}}{2} \int d k^{-}\left[A_{16}(\sigma-2 x)+2\left(B_{43}+x B_{46}\right)\right], \\
h_{T T}^{\perp}\left(x, k_{T}^{2}\right)= & -P^{+} \int d k^{-} A_{19} .
\end{aligned}
$$

\begin{tabular}{|c|c|c|c|c|c|c|}
\hline \multirow{3}{*}{ Hadron } & \multicolumn{6}{|c|}{ Quark } \\
\hline & \multicolumn{2}{|c|}{$\gamma^{\mathrm{i}}, 1, \mathrm{i} \gamma_{5}$} & \multicolumn{2}{|c|}{$\gamma^{+} \gamma_{5}$} & \multicolumn{2}{|c|}{$\sigma^{\mathrm{ij}}, \sigma^{-+}$} \\
\hline & T-even & T-odd & T-even & T-odd & T-even & T-odd \\
\hline $\mathrm{U}$ & {$[e]$} & & & & & \\
\hline $\mathrm{L}$ & & & & & {$\left[h_{L}\right]$} & \\
\hline $\mathrm{T}$ & & & $g_{T}$ & & & \\
\hline LL & {$\left[e_{L L}\right]$} & & & & & $* 3$ \\
\hline LT & $f_{L T}$ & & & $* 2$ & & \\
\hline TT & & & & & & \\
\hline
\end{tabular}

TABLE V. List of twist-3 quark collinear PDFs for a spin-1 hadron in terms of the hadron polarizations and the operator forms in the correlation functions. The square brackets [] indicate a chiral-odd distribution and the others are chiral-even ones. The LL and LT PDFs $\left(e_{L L}, f_{L T}\right)$ are new distributions found in this work. The asterisks $* 2$ and $* 3$ are explained in the main text. 
Because of the terms $A_{16}, \ldots, B_{52}$, these TMDs have properties of T-odd and $\chi$-odd as shown in Table IV. Here, the listed functions $f_{L T}, f_{T T}, h_{L T}$, and $h_{T T}$ are defined from $f_{L T}^{\prime}, f_{T T}^{\prime}, h_{L T}^{\prime}$, and $h_{T T}^{\prime}$, and $f_{L T}^{\perp}, f_{T T}^{\perp}, h_{L T}^{\perp}$, and $h_{T T}^{\perp}$ by the relation in Eq. (36).

These TMDs are integrated over the quark transverse momentum $\vec{k}_{T}$, twist-3 collinear PDFs exist as shown in Table V. In addition to the PDFs $e, g_{T}$, and $h_{L}$ in the nucleon, there are new twist-3 PDFs $e_{L L}$ and $f_{L T}$ for the spin-1 hadron. These two collinear PDFs are new functions found in this work by integrating the corresponding TMDs over $\vec{k}_{T}$. Here, the PDF $g_{T}$ is given by $g_{T}=\int d^{2} k_{T}\left[g_{T}^{\prime}-\right.$ $k_{T}^{2} /\left(2 M^{2}\right) g_{T}^{\perp}$ [ [34], and $f_{L T}(x)$ is defined by

$$
f_{L T}(x)=\int d^{2} k_{T} f_{L T}\left(x, k_{T}^{2}\right) .
$$

The asterisks $(* 2$ and $* 3)$ in Table $\mathrm{V}$ indicate the following in the same way with $* 1$. Because of the time-reversal invariance, the collinear PDFs $g_{L T}(x)$ and $h_{L L}(x)$ do not exist. However, the corresponding new collinear fragmentation functions $G_{L T}(z)$ and $H_{L L}(z)$ should exist [25].

\section{F. Twist-4 TMDs for a tensor-polarized spin-1 hadron}

The twist-4 TMDs were obtained in Ref. [34] for the spin-1/2 nucleon. Here, we list all the possible twist-4 quark TMDs for the tensor-polarized spin-1 hadron. The twist-4 TMDs for the tensor-polarized spin-1 hadron are defined in the correlation functions $\Phi^{\left[\gamma^{-}\right]}, \Phi^{\left[\gamma^{-} \gamma_{5}\right]}$, and $\Phi^{\left[\sigma^{i-}\right]}$ as

$$
\begin{aligned}
\Phi^{\left[\gamma^{-}\right]}= & \frac{M^{2}}{P^{+2}}\left[f_{3 L L}\left(x, k_{T}^{2}\right) S_{L L}-f_{3 L T}\left(x, k_{T}^{2}\right) \frac{S_{L T} \cdot k_{T}}{M}+f_{3 T T}\left(x, k_{T}^{2}\right) \frac{k_{T} \cdot S_{T T} \cdot k_{T}}{M^{2}}\right], \\
\Phi^{\left[\gamma^{-} \gamma_{5}\right]}= & \frac{M^{2}}{P^{+2}}\left[g_{3 L T}\left(x, k_{T}^{2}\right) \frac{S_{L T \mu} \varepsilon_{T}^{\mu \nu} k_{T \nu}}{M}+g_{3 T T}\left(x, k_{T}^{2}\right) \frac{S_{T T \mu \rho} k_{T}^{\rho} \varepsilon_{T}^{\mu \nu} k_{T \nu}}{M^{2}}\right], \\
\Phi^{\left[\sigma^{i-}\right]}= & \frac{M^{2}}{P^{+2}}\left[h_{3 L L}^{\perp}\left(x, k_{T}^{2}\right) S_{L L} \frac{k_{T}^{i}}{M}+h_{3 L T}^{\prime}\left(x, k_{T}^{2}\right) S_{L T}^{i}-h_{3 L T}^{\perp}\left(x, k_{T}^{2}\right) \frac{k_{T}^{i} S_{L T} \cdot k_{T}}{M^{2}}-h_{3 T T}^{\prime}\left(x, k_{T}^{2}\right) \frac{S_{T T}^{i j} k_{T j}}{M}\right. \\
& \left.+h_{3 T T}^{\perp}\left(x, k_{T}^{2}\right) \frac{k_{T} \cdot S_{T T} \cdot k_{T}}{M^{2}} \frac{k_{T}^{i}}{M}\right] .
\end{aligned}
$$

These relations are proportional to $1 /\left(P^{+}\right)^{2}$ as the twist- 4 functions.

The $f$-type TMDs are given by

$$
\begin{aligned}
f_{3 L L}\left(x, k_{T}^{2}\right)= & \frac{P^{+}}{6} \int d k^{-}\left\{\left[A_{14}+A_{15}(\sigma-x)\right] \tau_{x}\right. \\
& -2 A_{17}(\sigma-2 x)+2 B_{25} \tau_{x} \\
& \left.+2(\sigma-2 x)\left[2 B_{26}+B_{28}+B_{30}(\sigma-x)\right)\right] \\
& \left.+4\left(2 B_{27}+B_{29}-B_{32}\right)+4 B_{31}(\sigma-x)\right\}, \\
f_{3 L T}\left(x, k_{T}^{2}\right)= & -\frac{P^{+}}{2} \int d k^{-}\left\{\left[A_{14}+A_{15}(\sigma-x)\right](\sigma-2 x)\right. \\
& -A_{17}+2 B_{25}(\sigma-2 x)+2 B_{26}+B_{28} \\
& \left.+B_{30}(\sigma-x)\right\}, \\
& \frac{P^{+}}{2} \int d k^{-}\left[A_{14}+A_{15}(\sigma-x)+2 B_{25}\right] .
\end{aligned}
$$

Because of the terms $A_{14}, \ldots, B_{25}$, these TMDs have properties of T-even and $\chi$-even as shown in Table VI. The $g$-type TMDs are

$$
\begin{aligned}
g_{3 L T}\left(x, k_{T}^{2}\right)= & \frac{P^{+}}{4} \int d k^{-}\left[\left(A_{20}-2 B_{39}\right)(\sigma-2 x)\right. \\
& \left.+2 B_{33}-4 B_{40}\right], \\
g_{3 T T}\left(x, k_{T}^{2}\right)= & \frac{P^{+}}{2} \int d k^{-}\left(A_{20}-2 B_{39}\right) .
\end{aligned}
$$

These TMDs have the properties of T-odd and $\chi$-even as shown in Table VI. The $h$-type TMDs are

$$
\begin{aligned}
h_{3 L L}^{\perp}\left(x, k_{T}^{2}\right)= & \frac{P^{+}}{6} \int d k^{-}\left[-A_{16} \tau_{x}+2 A_{18}\right. \\
& +2 A_{19}(2 \sigma-3 x)-2\left(B_{41}-2 B_{47}\right)(\sigma-2 x) \\
& \left.+2 B_{46} \tau_{x}-4\left(B_{42}-2 B_{48}-B_{49}-B_{52}\right)\right], \\
h_{3 L T}^{\prime}\left(x, k_{T}^{2}\right)= & \frac{P^{+}}{4} \int d k^{-}\left\{\left[A_{18}+A_{19}(\sigma-x)\right](\sigma-2 x)\right. \\
& \left.+2\left[B_{49}(\sigma-2 x)+2 B_{50}+B_{51}+B_{52}(\sigma-x)\right]\right\}, \\
h_{3 L T}^{\perp}\left(x, k_{T}^{2}\right)= & -\frac{P^{+}}{2} \int d k^{-}\left[-A_{16}(\sigma-2 x)+A_{19}-B_{41}\right. \\
& \left.+2 B_{46}(\sigma-2 x)+2 B_{47}\right],
\end{aligned}
$$


TABLE VI. List of twist-4 quark TMDs for a spin-1 hadron in terms of the hadron polarizations and the operator forms in the correlation functions. The square brackets [] indicate chiral-odd distributions and the others are chiral-even ones. The LL, LT, and TT TMDs are new distributions found in this work.

\begin{tabular}{|c|c|c|c|c|c|c|}
\hline \multirow{3}{*}{ Hadron } & \multicolumn{6}{|c|}{ Quark } \\
\hline & \multicolumn{2}{|c|}{$\gamma^{-}$} & \multicolumn{2}{|c|}{$\gamma^{-} \gamma_{5}$} & \multicolumn{2}{|c|}{$\sigma^{\mathrm{i}-}$} \\
\hline & T-even & T-odd & T-even & T-odd & T-even & T-odd \\
\hline $\mathrm{U}$ & $f_{3}$ & & & & & {$\left[h_{3}^{\perp}\right]$} \\
\hline $\mathrm{L}$ & & & $g_{3 L}$ & & {$\left[h_{3 L}^{\perp}\right]$} & \\
\hline $\mathrm{T}$ & & $f_{3 T}^{\perp}$ & $g_{3 T}$ & & {$\left[h_{3 T}\right],\left[h_{3 T}^{\perp}\right]$} & \\
\hline LL & $f_{3 L L}$ & & & & & {$\left[h_{3 L L}^{\perp}\right]$} \\
\hline LT & $f_{3 L T}$ & & & $g_{3 L T}$ & & {$\left[h_{3 L T}\right],\left[h_{3 L T}^{\perp}\right]$} \\
\hline TT & $f_{3 T T}$ & & & $g_{3 T T}$ & & {$\left[h_{3 T T}\right],[h \stackrel{\perp}{3 T T}]$} \\
\hline
\end{tabular}

$h_{3 T T}^{\prime}\left(x, k_{T}^{2}\right)=-\frac{P^{+}}{2} \int d k^{-}\left[A_{18}+A_{19}(\sigma-x)+2 B_{49}\right]$,

$h_{3 T T}^{\perp}\left(x, k_{T}^{2}\right)=-\frac{P^{+}}{2} \int d k^{-}\left[A_{16}-2 B_{46}\right]$.

These TMDs have the properties of T-odd and $\chi$-odd as shown in Table VI. Here, the functions $h_{3 T}, h_{3 L T}$, and $h_{3 T T}$ are defined from $h_{3 T}^{\prime}, h_{3 L T}^{\prime}$, and $h_{3 T T}^{\prime}$ and $h_{3 T}^{\perp}, h_{3 L T}^{\perp}$, and $h_{3 T T}^{\perp}$ by the relation in Eq. (36).

These twist-4 TMDs are integrated over $\vec{k}_{T}$ and they become the collinear PDFs as shown in Table VII. Most distributions vanish after the integrations. The only twist- 4 PDF which is specific to the tensor-polarized spin- 1 hadron is $f_{3 L L}$, in addition to $f_{3}, g_{3 L}$, and $h_{3 L}$ which exist also for the spin- $1 / 2$ nucleon. The asterisk $(* 4)$ in Table VII indicates that $h_{3 L T}(x)$ does not exist; however, the corresponding new collinear fragmentation function $H_{3 L T}(z)$ should exist because the time-reversal invariance does not have to be imposed [25].

TABLE VII. List of twist-4 quark collinear PDFs for a spin-1 hadron in terms of the hadron polarizations and the operator forms in the correlation functions. The square bracket [] indicates a chiral-odd distribution and the others are chiral-even ones. The LL PDF $\left(f_{3 L L}\right)$ is a new distribution found in this work. The asterisk $* 4$ is explained in the main text.

\begin{tabular}{|c|c|c|c|c|c|c|}
\hline \multirow{3}{*}{ Hadron } & \multicolumn{6}{|c|}{ Quark } \\
\hline & \multicolumn{2}{|c|}{$\gamma^{-}$} & \multicolumn{2}{|c|}{$\gamma^{-} \gamma_{5}$} & \multicolumn{2}{|c|}{$\sigma^{\mathrm{i}-}$} \\
\hline & T-even & T-odd & T-even & T-odd & T-even & T-odd \\
\hline $\mathrm{U}$ & $f_{3}$ & & & & & \\
\hline L & & & $g_{3 L}$ & & & \\
\hline $\begin{array}{l}\text { T } \\
\text { LL }\end{array}$ & $f_{3 L L}$ & & & & {$\left[h_{3 T}\right]$} & \\
\hline $\begin{array}{l}\text { LT } \\
\text { TT }\end{array}$ & & & & & & $* 4$ \\
\hline
\end{tabular}

\section{G. Summary on new TMDs and possible new fragmentation functions}

We found that there are 40 TMDs in total for the tensorpolarized spin- 1 hadron, and this number is equal to the one of the expansion terms in Eq. (20), and they are expressed by the coefficients $A_{i}$ and $B_{i}$. The TMDs are T-odd if they are associated with the gamma matrices $\gamma^{\mu} \gamma_{5}$ and $\sigma^{\mu \nu}$ in the tensor-polarized case, so that there are 24 T-odd TMDs. In addition, there are 16 T-even TMDs on the tensor polarizations. If the gauge link were neglected in the correlation function, all the T-odd TMDs do not exist due to the timereversal invariance. The ten twist-2 TMDs were studied in Ref. [40], so we found 30 new TMDs in the twist-3 and twist-4 parts mainly associated with the light cone vector $n$ and the tensor polarizations as listed in Tables IV and VI.

The same discussions can be made for the transversemomentum-dependent fragmentation functions of spin-1 hadrons by the replacements of the kinematical variables and the function notations as [40]

Kinematical variables: $x, k_{T}, S, T, M, n, \gamma^{+}, \sigma^{i+}$,

TMD distribution functions: $f, g, h, e$

\section{$\Downarrow$}

Kinematical variables: $z, k_{T}, S_{h}, T_{h}, M_{h}, \bar{n}, \gamma^{-}, \sigma^{i-}$, TMD fragmentation functions: $D, G, H, E$.

Therefore, new fragmentation functions exist for spin-1 hadrons in addition to the fragmentation functions of the spin- $1 / 2$ nucleon by these simple replacements in Tables II-VII. Here, $S_{h}$ and $T_{h}$ are spin-vector and tensor polarizations of the hadron $h$, and $M_{h}$ is its mass. The variable $z$ is the momentum fraction given by $P_{h}^{-}=z k^{-}$. As explained by the asterisks $(* 1-4)$ in the collinear PDF tables, there are the collinear fragmentation functions $H_{1 L T}(z), G_{L T}(z), H_{L L}(z)$, and $H_{3 L T}(z)$, although their corresponding functions $h_{1 L T}(x), g_{L T}(x), h_{L L}(x)$, and $h_{3 L T}(x)$ vanish due to the time-reversal invariance.

\section{H. Integral relations in T-odd TMDs}

If we integrate the $k_{T}$-dependent correlation function $\Phi\left(x, k_{T}, T\right)$ over $k_{T}$, the T-odd terms should vanish on account of time-reversal invariance $\left(\int d^{2} k_{T} \Phi_{\text {T-odd }}=0\right)$ $[34,38,58]$, so that the following sum rules should be satisfied:

$$
\begin{aligned}
& \int d^{2} k_{T} h_{1 L T}\left(x, k_{T}^{2}\right)=0, \\
& \int d^{2} k_{T} g_{L T}\left(x, k_{T}^{2}\right)=0, \\
& \int d^{2} k_{T} h_{L L}\left(x, k_{T}^{2}\right)=0, \\
& \int d^{2} k_{T} h_{3 L T}\left(x, k_{T}^{2}\right)=0 .
\end{aligned}
$$


In the twist-2, although the collinear PDF $h_{1 L T}(x)$ vanishes, its corresponding fragmentation function $H_{1 L T}(z)$ exists as noted in Ref. [25] as the function $\hat{h}_{\overline{1}}$. These T-odd terms are proportional to $\left(k_{T}\right)^{0}$ or $\left(k_{T}\right)^{2}$ in the correlation functions $\Phi^{[\Gamma]}$. The terms with $\left(k_{T}\right)^{1}$ vanish and the term $k_{T} \cdot S_{T T} \cdot k_{T}$ also vanishes after integrations, so there is no similar sum rule for other TMDs. Similar sum rules exist for the TMDs $f_{1 T}^{\perp}$ and $h$ in the spin-1/2 part as shown in Eqs. (22) and (23) of Ref. [38]. We may note that such a sum rule does not exist for the fragmentation functions since the time-reversal invariance cannot be imposed on the fragmentation functions, which contain the out-state $\left|P_{h}, S_{h}, X\right\rangle$ in its definition $[25,34,40,59]$.

\section{SUMMARY}

The possible TMDs were investigated for tensorpolarized spin-1 hadrons by the complete decomposition of the quark correlation function including the light cone vector $n$ in this work. We found the 32 new terms which are dependent mainly on the vector $n$ in decomposing the correlation function, so that there are totally 40 independent terms in the tensor-polarized correlation function. Furthermore, the tensor-polarized TMDs were studied up to twist-4 level for the spin-1 hadron, and the 40 TMDs are found in association with the tensor polarization. There exist ten TMDs in the twist- 2 case. Due to the existence of the new terms $\left(B_{20-52}\right)$, the twist-2 TMD expressions of $f_{1 L L}, f_{1 L T}, g_{1 L T}, h_{1 L L}^{\perp}, h_{1 L T}, h_{1 L T}^{\perp}$ in terms of the expansion coefficients $A_{i}$ are modified. All the twist-3 and 4 TMDs (the following 30 TMDs) on the tensor-polarized spin-1 hadron,
Twist-3 TMD: $f_{L L}^{\perp}, e_{L L}, f_{L T}, f_{L T}^{\perp}, e_{1 T}, e_{1 T}^{\perp}, f_{T T}, f_{T T}^{\perp}$, $e_{T T}, e_{T T}^{\perp}, g_{L L}^{\perp}, g_{L T}, g_{L T}^{\perp}, g_{T T}, g_{T T}^{\perp}, h_{1 L}$, $h_{L T}, h_{L T}^{\perp}, h_{T T}, h_{T T}^{\perp}$,

Twist-4 TMD: $f_{3 L L}, f_{3 L T}, f_{3 T T}, g_{3 L T}, g_{3 T T}, h_{3 L L}^{\perp}, h_{3 L T}$, $h_{3 L T}^{\perp}, h_{3 T T}, h_{3 T T}^{\perp}$,

are new functions we found in this work. We also found new sum rules for the TMDs as $\int d^{2} k_{T} g_{L T}=\int d^{2} k_{T} h_{L L}=$ $\int d^{2} k_{T} h_{3 L L}=0$. Integrating these new TMDs, we found the collinear PDFs,

Twist-3 PDF: $e_{L L}, f_{L T}$,

Twist-4 PDF: $f_{3 L L}$,

in this work. In addition, we explained that the corresponding transverse-momentum-dependent fragmentation functions exist for the tensor-polarized spin-1 hadrons.

Recently, the T-odd TMDs attract considerable attention since they are related to single spin asymmetries in the proton reactions. The T-odd TMDs in the spin-1 deuteron are also interesting to be investigated in future. Since there are projects to investigate the structure functions of the polarized spin-1 deuteron at JLab, Fermilab, NICA, and EIC, we hope that these new structure functions will be experimentally investigated in future.

\section{ACKNOWLEDGMENTS}

The authors thank A. Bacchetta for suggestions for understanding Ref. [40], and they thank P. J. Mulders for usual comments. The work of S. K. was partially supported by Japan Society for the Promotion of Science Grants-in-Aid for Scientific Research (KAKENHI) Grant No. 19K03830. The work of Q.-T. S. was supported by the National Natural Science Foundation of China under Grant No. 12005191 and the Academic Improvement Project of Zhengzhou University.
[1] J. Ashman et al. (European Muon Collaboration), Phys. Lett. B 206, 364 (1988).

[2] For review, see S. E. Kuhn, J.-P. Chen, and E. Leader, Prog. Part. Nucl. Phys. 63, 1 (2009); A. Deur, S. J. Brodsky, and G. F. de Teramond, Rep. Prog. Phys. 82, 076201 (2019), and references therein.

[3] K. Goeke, M. V. Polyakov, and M. Vanderhaeghen, Prog. Part. Nucl. Phys. 47, 401 (2001); M. Diehl, Phys. Rep. 388, 41 (2003); X. Ji, Annu. Rev. Nucl. Part. Sci. 54, 413 (2004); A. V. Belitsky and A. V. Radyushkin, Phys. Rep. 418, 1 (2005); S. Boffi and B. Pasquini, Riv. Nuovo Cimento 30, 387 (2007); M. Diehl and P. Kroll, Eur. Phys. J. C 73, 2397 (2013); D. Mueller, Few Body Syst. 55, 317 (2014); S. Wallon, Doctoral school lecture notes on courses ED-107 and ED-517, Université Paris Sud (2014), unpublished, http://pperso.th.u-psud.fr/page_perso/ Wallon/cours/exclusif.pdf; K. Kumericki, S. Liuti, and H. Moutarde, Eur. Phys. J. A 52, 157 (2016); H. Moutarde,
P. Sznajder, and J. Wagner, Eur. Phys. J. C 78, 890 (2018).

[4] S. Kumano, Q.-T. Song, and O. V. Teryaev, Phys. Rev. D 97, 014020 (2018).

[5] U. D’Alesio and F. Murgia, Prog. Part. Nucl. Phys. 61, 394 (2008); V. Barone, F. Bradamante, and A. Martin, Prog. Part. Nucl. Phys. 65, 267 (2010); C. A. Aidala, S. D. Bass, D. Hasch, and G. K. Mallot, Rev. Mod. Phys. 85, 655 (2013); M. G. Perdekamp and F. Yuan, Annu. Rev. Nucl. Part. Sci. 65, 429 (2015); R. Angeles-Martinez et al., Acta Phys. Pol. B 46, 2501 (2015).

[6] For recent progress, see T. Altinoluk, R. Boussarie, C. Marquet, and P. Taels, J. High Energy Phys. 07 (2020) 143; H. Fujii, C. Marquet, and K. Watanabe, J. High Energy Phys. 12 (2020) 181.

[7] Y. Aharonov and D. Bohm, Phys. Rev. 115, 485 (1959); M. Peshkin and A. Tonomura, The Aharonov-Bohm Effect, Lecture Notes in Physics (Springer-Verlag, Berlin, 1989), 
Vol. 340; Y. Aharonov and D. Rohrlich, Quantum Paradoxes (Wiley-VCH Verlag GmbH \& Co. KGaA, Weinheim, 2005).

[8] P. J. Mulders, Proposal No 320389W for the project, Quantum Chromodynamics at Work (2012), http://www .nat.vu.nl/ mulders/workplan-ERC.pdf.

[9] T. C. Rogers and P. J. Mulders, Phys. Rev. D 81, 094006 (2010); A. Adare et al. (PHENIX Collaboration), Phys. Rev. D 95, 072002 (2017); J. Qiu, Workshop on Progress on Hadron Structure Functions in 2018, Nov. 18-19, 2018, KEK, Tsukuba, Japan (2018), https://j-parc-th.kek.jp/ workshops/2018/11-18/.

[10] L. L. Frankfurt and M. I. Strikman, Nucl. Phys. A405, 557 (1983).

[11] P. Hoodbhoy, R. L. Jaffe, and A. Manohar, Nucl. Phys. B312, 571 (1989).

[12] R. L. Jaffe and A. Manohar, Phys. Lett. B 223, 218 (1989); J. P. Ma, C. Wang, and G. P. Zhang, arXiv:1306.6693 (unpublished); M. Jones et al., A letter of intent to Jefferson Lab PAC 44, LOI12-16-006 (2016).

[13] A. Airapetian et al. (HERMES Collaboration), Phys. Rev. Lett. 95, 242001 (2005).

[14] W. Cosyn, Y.-B. Dong, S. Kumano, and M. Sargsian, Phys. Rev. D 95, 074036 (2017).

[15] W. Cosyn and C. Weiss, Phys. Rev. C 102, 065204 (2020).

[16] F. E. Close and S. Kumano, Phys. Rev. D 42, 2377 (1990); S. Kumano, J. Phys. Conf. Ser. 543, 012001 (2014).

[17] S. Kumano, Phys. Rep. 303, 183 (1998); G. T. Garvey and J.-C. Peng, Prog. Part. Nucl. Phys. 47, 203 (2001); J.-C. Peng and J.-W. Qiu, Prog. Part. Nucl. Phys. 76, 43 (2014).

[18] J.-P. Chen et al., Proposal to Jefferson Lab PAC-38 (PR1211-110) (2011), https://www.jlab.org/exp_prog/proposals/ 11/PR12-11-110.pdf.

[19] Fermilab E1039 experiment, Letter of intent Report No. P1039, 2013, https://www.fnal.gov/directorate/program_ planning/June2013PACPublic/P-1039_LOI_polarized_DY .pdf.

[20] D. Keller, D. Crabb, and D. Day, Nucl. Instrum. Methods Phys. Res., Sect. A 981, 164504 (2020).

[21] S. Hino and S. Kumano, Phys. Rev. D 59, 094026 (1999); 60, 054018 (1999).

[22] S. Kumano and Q. T. Song, Phys. Rev. D 94, 054022 (2016).

[23] S. Kumano, Phys. Rev. D 82, 017501 (2010).

[24] E. R. Berger, F. Cano, M. Diehl, and B. Pire, Phys. Rev. Lett. 87, 142302 (2001); W. Cosyn and B. Pire, Phys. Rev. D 98, 074020 (2018); B.-D. Sun and Y.-B. Dong, Phys. Rev. D 96, 036019 (2017); 101, 096008 (2020).

[25] X. Ji, Phys. Rev. D 49, 114 (1994).

[26] S. Kumano and Q. T. Song, Phys. Rev. D 101, 054011 (2020); 101, 094013 (2020).

[27] For the Spin Physics Detector (SPD) project at NICA, see http://spd.jinr.ru/; A. Arbuzov et al., arXiv:2011.15005.

[28] J. P. Ralston and D. E. Soper, Nucl. Phys. B152, 109 (1979).

[29] R. D. Tangerman and P. J. Mulders, Phys. Rev. D 51, 3357 (1995).

[30] P. J. Mulders and R. D. Tangerman, Nucl. Phys. B461, 197 (1996); 484, 538(E) (1997).
[31] R. D. Tangerman, Ph.D. thesis, Free University Amsterdam, 1996.

[32] K. Goeke, A. Metz, P. V. Pobylitsa, and M. V. Polyakov, Phys. Lett. B 567, 27 (2003).

[33] A. Bacchetta, P. J. Mulders, and F. Pijlman, Phys. Lett. B 595, 309 (2004).

[34] K. Goeke, A. Metz, and M. Schlegel, Phys. Lett. B 618, 90 (2005).

[35] D. Boer and P. J. Mulders, Phys. Rev. D 57, 5780 (1998).

[36] R. Kundu and A. Metz, Phys. Rev. D 65, 014009 (2001).

[37] S. Wandzura and F. Wilczek, Phys. Lett. 72B, 195 (1977).

[38] A. Metz, P. Schweitzer, and T. Teckentrup, Phys. Lett. B 680, 141 (2009).

[39] A. Accardi, A. Bacchetta, W. Melnitchouk, and M. Schlegel, J. High Energy Phys. 11 (2009) 093.

[40] A. Bacchetta and P. Mulders, Phys. Rev. D 62, 114004 (2000). In expressing the twist-2 TMDs in this paper, their notations of Appendix $\mathrm{C}$ are used by noting their convention, $a_{i}($ Ref. $[40])=-a_{i}($ in this paper $)=a^{i}$.

[41] P. Mulders and J. Rodrigues, Phys. Rev. D 63, 094021 (2001).

[42] Y. Ninomiya, W. Bentz, and I. C. Cloet, Phys. Rev. C 96, 045206 (2017).

[43] D. Boer, P. J. Mulders, and F. Pijlman, Nucl. Phys. B667, 201 (2003).

[44] J. C. Collins and D. E. Soper, Nucl. Phys. B194, 445 (1982); J. C. Collins, Phys. Lett. B 536, 43 (2002).

[45] X. Ji and F. Yuan, Phys. Lett. B 543, 66 (2002).

[46] A. V. Belitsky, X. Ji, and F. Yuan, Nucl. Phys. B656, 165 (2003).

[47] S. J. Brodsky, D. S. Hwang, and I. Schmidt, Phys. Lett. B 530, 99 (2002); Nucl. Phys. B642, 344 (2002).

[48] E. Leader, Spin in Particle Physics (Cambridge University Press, Cambridge, United Kingdom, 2001).

[49] S. Weinberg, The Quantum Theory of Fields (Cambridge University Press, Cambridge, United Kingdom, 1995), Vol. I.

[50] J. D. Bjorken and S. D. Drell, Relativistic Quantum Fields (McGraw-Hill, New York, 1965).

[51] C. Itzykson and J.-B. Zuber, Quantum Field Theory (McGraw-Hill Inc., New York, 1980).

[52] V. Barone and R. G. Ratcliffe, Transverse Spin Physics (World Scientific, Singapore, 2003).

[53] J. Qiu and G. Sterman, Phys. Rev. Lett. 67, 2264 (1991).

[54] J. Collins, Phys. Lett. B 536, 43 (2002).

[55] L. Adamczyk et al. (STAR Collaboration), Phys. Rev. Lett. 116, 132301 (2016).

[56] M. Aghasyan et al. (COMPASS Collaboration), Phys. Rev. Lett. 119, 112002 (2017).

[57] R. L. Jaffe, in Lectures on $Q C D$, edited by F. Lenz, H. W. Grießhammer, and D. Stoll (Springer-Verlag, Berlin, Heidelberg, 1997), pp. 178-249.

[58] A. Bacchetta, M. Diehl, K. Goeke, A. Metz, P. J. Mulders, and M. Schlegel, J. High Energy Phys. 02 (2007) 093.

[59] A. Metz and A. Vossen, Prog. Part. Nucl. Phys. 91, 136 (2016).

Correction: The first terms with an integral in the abstract and in the first paragraph of Sec. IV contained errors and have been fixed. 